UDC 536.75, 538.9, 548:537.621, 538.955-405;

PACS 05.50.+q, 05.70.Ce, 64.60.Fr, 75.10.Hk

\title{
CALCULATION METHOD FOR THE THREE-DIMENSIONAL ISING FERROMAGNET THERMODYNAMICS WITHIN THE FRAMES OF $\rho^{6}$ MODEL
}

\author{
M.P.KozlovskiI, I.V.Pylyuk, V.V.DukhoviI \\ Institute for Condensed Matter Physics \\ of the Ukrainian National Academy of Sciences, \\ 1 Svientsitskii St., UA-290011 Lviv, Ukraine
}

Received August 19, 1997

\begin{abstract}
Calculation of thermodynamic functions of the three-dimensional Ising ferromagnet above and below critical temperature is performed in the approximation of sixfold basis distribution ( $\rho^{6}$ model $)$. Comparison with the results for the $\rho^{4}$ model indicates that dependence of the thermodynamic functions on the renormalization group parameter $s$ becomes weaker. The optimal interval of the renormalization group parameter values is determined.
\end{abstract}

\section{Introduction}

Significant results in the description of the system thermodynamic properties in the vicinity of the transition point have been obtained by means of the collective variables (CV) approach. The method of deriving explicit expressions for the thermodynamic and correlation functions of the threedimensional Ising model at temperatures both above and below critical temperature $T_{c}$ has been suggested within this approach. The calculations are performed with a non-Gaussian measure density. The measure is represented as an exponential function of CV, the argument of which contains, along with the quadratic term, higher powers of the variable with the corresponding interaction constants. The simplest non-Gaussian measure density is the quartic one ( $\rho^{4}$ model) with the second and the fourth powers of the variable in the exponent. Then the sixfold measure goes containing the sixth power of the variable $\left(\rho^{6}\right.$ model $)$, and so forth.

The results of the theory depend on the renormalization group (RG) parameter $s$ due to an approximation of the Ising model partition function calculation using the non-Gaussian measure densities. This dependence decreases essentially if the non-Gaussian measure density becomes more complicated. Calculations of the correlation length critical exponent $\nu$ within the $\rho^{2 m}$ models with $m=2,3,4,5$ confirm this statement [1-3]. It has been established that the $\rho^{6}$ model provides an adequate description of the Ising model critical behaviour, in particular, the critical exponents, at the RG parameter values in the interval $2 \leqslant s \leqslant 4$.

Investigation of the $\rho^{6}$ model within the numerical realization of the $\mathrm{CV}$ method has been performed in [4]. Analytical derivation of the explicit expressions for the $\rho^{6}$ model thermodynamic functions is the subject of the present paper. The foundations for such kind of investigations have

(C) M.P.Kozlovskii, I.V.Pylyuk, V.V.Dukhovii, 1997

ISSN 0452-9910. Condensed Matter Physics 1997 No 11 (17-49) 
been developed in [5-9], where the quartic distribution was used as a basis measure.

\section{General relations}

The partition function of the three-dimensional Ising model within the sixfold measure density is given by

$$
\begin{aligned}
& Z=2^{N} \int \exp \left[\frac{1}{2} \sum_{k \leqslant B} \beta \tilde{\Phi}(k) \rho_{\boldsymbol{k}} \rho_{-\boldsymbol{k}}+2 \pi i \sum_{k \leqslant B} \omega_{\boldsymbol{k}} \rho_{\boldsymbol{k}}+\sum_{n=1}^{3}(2 \pi i)^{2 n} \times\right. \\
& \left.\times N^{1-n} \frac{1}{(2 n) !} \sum_{k_{1}, \ldots, k_{2 n} \leqslant B} \mathcal{M}_{2 n} \omega_{\boldsymbol{k}_{1}} \cdots \omega_{\boldsymbol{k}_{2 n}} \delta_{\boldsymbol{k}_{1}+\cdots+\boldsymbol{k}_{2 n}}\right](\mathrm{d} \omega)^{N}(\mathrm{~d} \rho)^{N},
\end{aligned}
$$

where $\mathcal{M}_{2}=1, \mathcal{M}_{4}=-2, \mathcal{M}_{6}=16, \tilde{\Phi}(k)=\tilde{\Phi}(0)\left(1-2 b^{2} k^{2}\right), \beta=(k T)^{-1}$ is the inverse temperature, $b$ is the effective interaction radius of the potential $\Phi(r)=A \exp (-r / b), \tilde{\Phi}(0)=8 \pi A(b / c)^{3}$. Integrating (1.1) over $\rho_{\boldsymbol{k}}$ and $\omega_{\boldsymbol{k}}$ with the indices $B^{\prime}<|\boldsymbol{k}| \leqslant B(B=\pi / c, c$ is the simple cubic lattice constant), we get an expression for the partition function of the $\rho^{6}$ model:

$$
\begin{aligned}
Z= & 2^{N} 2^{\frac{N^{\prime}-1}{2}} \mathrm{e}^{a^{\prime}{ }_{0} N^{\prime}} \int \exp \left[-\frac{1}{2} \sum_{k \leq B^{\prime}} d^{\prime}(k) \rho_{\boldsymbol{k}} \rho_{-\boldsymbol{k}}-\right. \\
& \left.-\sum_{l=2}^{3} \frac{1}{(2 l) !}\left(N^{\prime}\right)^{1-l} \sum_{k_{1}, \ldots, k_{2 l} \leqslant B^{\prime}} a_{2 l} \rho_{\boldsymbol{k}_{1}} \cdots \rho_{\boldsymbol{k}_{2 l}} \delta_{\boldsymbol{k}_{1}+\cdots+\boldsymbol{k}_{2 l}}\right](\mathrm{d} \rho)^{N^{\prime}} .
\end{aligned}
$$

Here $N^{\prime}=N s_{0}^{-3}, s_{0}=B / B^{\prime}=\pi \sqrt{2} b / c$,

$$
d^{\prime}(k)=a_{2}^{\prime}-\beta \tilde{\Phi}(k) .
$$

Coefficients $a_{2 l}^{\prime}$ depend on the ratio $b / c$ and are given by the relations

$$
\begin{aligned}
& a_{0}^{\prime}=\ln Q(\mathcal{M}), \quad Q(\mathcal{M})=\left(12 s_{0}^{3}\right)^{1 / 4} \pi^{-1} I_{0}\left(\eta^{\prime}, \xi^{\prime}\right), \\
& a_{2}^{\prime}=\left(12 s_{0}^{3}\right)^{1 / 2} \mathcal{F}_{2}\left(\eta^{\prime}, \xi^{\prime}\right), \\
& a_{4}^{\prime}=12 s_{0}^{3} C\left(\eta^{\prime}, \xi^{\prime}\right), \\
& a_{6}^{\prime}=\left(12 s_{0}^{3}\right)^{3 / 2} N\left(\eta^{\prime}, \xi^{\prime}\right),
\end{aligned}
$$

where the quantities $\eta^{\prime}=\sqrt{3} s_{0}^{3 / 2}, \xi^{\prime}=\frac{8 \sqrt{3}}{15 s_{0}^{3 / 2}}$ are the arguments, and special functions $C\left(\eta^{\prime}, \xi^{\prime}\right)$ and $N\left(\eta^{\prime}, \xi^{\prime}\right)$ read

$$
\begin{aligned}
C\left(\eta^{\prime}, \xi^{\prime}\right) & =-\mathcal{F}_{4}\left(\eta^{\prime}, \xi^{\prime}\right)+3 \mathcal{F}_{2}^{2}\left(\eta^{\prime}, \xi^{\prime}\right), \\
N\left(\eta^{\prime}, \xi^{\prime}\right) & =\mathcal{F}_{6}\left(\eta^{\prime}, \xi^{\prime}\right)-15 \mathcal{F}_{4}\left(\eta^{\prime}, \xi^{\prime}\right) \mathcal{F}_{2}\left(\eta^{\prime}, \xi^{\prime}\right)+30 \mathcal{F}_{2}^{3}\left(\eta^{\prime}, \xi^{\prime}\right) .
\end{aligned}
$$

Here $\mathcal{F}_{2 l}\left(\eta^{\prime}, \xi^{\prime}\right)=\frac{I_{2 l}\left(\eta^{\prime}, \xi^{\prime}\right)}{I_{0}\left(\eta^{\prime}, \xi^{\prime}\right)}, I_{2 l}\left(\eta^{\prime}, \xi^{\prime}\right)=\int_{0}^{\infty} t^{2 l} \mathrm{e}^{-\eta^{\prime} t^{2}-t^{4}-\xi^{\prime} t^{6}} \mathrm{~d} t$.

Using the method of layer-by-layer integration of the partition function in the phase space of $\mathrm{CV}$, developed in [10], one can reduce (1.2) to the form:

$$
Z=2^{N} 2^{\frac{N_{n+1}-1}{2}} Z_{0} Z_{1} \ldots Z_{n}\left(Q\left(P_{n}\right)\right)^{N_{n+1}} \int w_{6}^{(n+1)}(\rho)(\mathrm{d} \rho)^{N_{n+1}},
$$


where $N_{n}=N^{\prime} s^{-3 n}$,

$$
\begin{aligned}
& Z_{0}=[Q(\mathcal{M}) Q(d)]^{N^{\prime}}, Z_{1}=\left[Q(P) Q\left(d_{1}\right)\right]^{N_{1}}, \ldots, \\
& Z_{n}=\left[Q\left(P_{n-1}\right) Q\left(d_{n}\right)\right]^{N_{n}}, \\
& Q\left(P_{n}\right)=\frac{1}{\pi}\left(s^{3} \frac{a_{4}^{(n)}}{C\left(h_{n}, \alpha_{n}\right)}\right)^{1 / 4} I_{0}\left(\eta_{n}, \xi_{n}\right), \\
& Q\left(d_{n}\right)=2\left(24 / a_{4}^{(n)}\right)^{1 / 4} I_{0}\left(h_{n}, \alpha_{n}\right) .
\end{aligned}
$$

Hereafter the arguments $h_{n}, \alpha_{n}$ are called basic:

$$
h_{n}=d_{n}\left(B_{n+1}, B_{n}\right)\left(6 / a_{4}^{n}\right)^{1 / 2}, \quad \alpha_{n}=\frac{\sqrt{6}}{15} a_{6}^{(n)} /\left(a_{4}^{(n)}\right)^{3 / 2} .
$$

The effective measure density of the $\mathrm{n}$-th phase layer $w_{6}^{(n)}(\rho)$ has the form:

$$
\begin{aligned}
w_{6}^{(n)}(\rho) & =\exp \left[-\frac{1}{2} \sum_{k \leq B_{n}} d_{n}(k) \rho_{\boldsymbol{k}} \rho_{-\boldsymbol{k}}-\right. \\
& \left.-\sum_{l=2}^{3} \frac{1}{(2 l) !} N_{n}^{1-l} \sum_{k_{1}, \ldots, k_{2 l} \leqslant B_{n}} a_{2 l}^{(n)} \rho_{\boldsymbol{k}_{1}} \cdots \rho_{\boldsymbol{k}_{2 l}} \delta_{\boldsymbol{k}_{1}+\cdots+\boldsymbol{k}_{2 l}}\right] .
\end{aligned}
$$

Here $B_{n}=B^{\prime} s^{-n}$. The intermediate variables $\eta_{n}, \xi_{n}$ are functions of $h_{n}$ and $\alpha_{n}:$

$$
\begin{aligned}
& \eta_{n}=\sqrt{6} s^{3 / 2} \mathcal{F}_{2}\left(h_{n}, \alpha_{n}\right)\left[C\left(h_{n}, \alpha_{n}\right)\right]^{-1 / 2}, \\
& \xi_{n}=\frac{\sqrt{6}}{15} s^{-3 / 2} N\left(h_{n}, \alpha_{n}\right)\left[C\left(h_{n}, \alpha_{n}\right)\right]^{-3 / 2} .
\end{aligned}
$$

The form of the special functions $C\left(h_{n}, \alpha_{n}\right), N\left(h_{n}, \alpha_{n}\right)$ is given by (1.5).

Coefficients $d_{n}\left(B_{n+1}, B_{n}\right), a_{4}^{(n)}, a_{6}^{(n)}$ are related to the coefficients of the $(n+1)$ th layer by recurrent relations (RR) [11-13]. The solutions of these relations [13] are used in the calculation of the system thermodynamic characteristics.

\section{Thermodynamic functions of the $\rho^{6}$ model in the regions of crit- ical and limit Gaussian regimes (CR and LGR) above $T_{c}$}

It is convenient to rewrite the model partition function as [14]

$$
Z=2^{N} Z_{C R} Z_{L G R}
$$

Let us consider $Z_{C R}$ given by

$$
Z_{C R}=\prod_{n=0}^{m_{\tau}}\left[\frac{2}{\pi}\left(\frac{24}{C\left(\eta_{n-1}, \xi_{n-1}\right)}\right)^{1 / 4} I_{0}\left(h_{n}, \alpha_{n}\right) I_{0}\left(\eta_{n-1}, \xi_{n-1}\right)\right]^{N_{n}} .
$$

It should be mentioned that in (2.2) $\eta_{-1} \equiv \eta^{\prime}, \quad \xi_{-1} \equiv \xi^{\prime}$ at $n=0$. We represent the right-hand side (RHS) of (2.2) in the form of an explicit dependence on the phase layer number $n$ in order to calculate $Z_{C R}$. 
In the $\mathrm{CR}$ region, the basic $h_{n}, \alpha_{n}$ and intermediate $\eta_{n}, \xi_{n}$ arguments are close to their values at the fixed point. Therefore, functions of these arguments can be written as power series of deviations of basic arguments from their values at the fixed point (see $[15,16]$ ). Using the obtained representations for $I_{0}\left(h_{n}, \alpha_{n}\right), I_{0}\left(\eta_{n-1}, \xi_{n-1}\right), C\left(\eta_{n-1}, \xi_{n-1}\right)$, we determine from (2.2) the partial free energy corresponding to the $n$-th phase layer:

$$
\begin{aligned}
F_{n}= & -k T N_{n}\left\{f_{C R}^{(0)}+\varphi_{1}\left(h_{n-1}-h^{(0)}\right)+\varphi_{2}\left(\alpha_{n-1}-\alpha^{(0)}\right)+\right. \\
& +\varphi_{3}\left(h_{n}-h^{(0)}\right)+\varphi_{4}\left(\alpha_{n}-\alpha^{(0)}\right)+\varphi_{1}^{\prime}\left(h_{n-1}-h^{(0)}\right)^{2}+ \\
& +\varphi_{2}^{\prime}\left(\alpha_{n-1}-\alpha^{(0)}\right)^{2}+\varphi_{3}^{\prime}\left(h_{n}-h^{(0)}\right)^{2}+\varphi_{4}^{\prime}\left(\alpha_{n}-\alpha^{(0)}\right)^{2}+ \\
& \left.+\varphi_{5}^{\prime}\left(h_{n-1}-h^{(0)}\right)\left(\alpha_{n-1}-\alpha^{(0)}\right)+\varphi_{6}^{\prime}\left(h_{n}-h^{(0)}\right)\left(\alpha_{n}-\alpha^{(0)}\right)\right\} \\
f_{C R}^{(0)}= & \ln \left(\frac{2(24)^{1 / 4}}{\pi}\right)-\frac{1}{4} \ln \mathcal{P}_{40}+\ln I_{0}^{*}+\ln I_{0}^{* *}, \\
\varphi_{m}= & b_{m}+\mathcal{P}_{4 m} / 4, m=1,2, \\
\varphi_{3}= & -\mathcal{F}_{2}^{*}, \varphi_{4}=-\mathcal{F}_{6}^{*}, \\
\varphi_{m}^{\prime}= & b_{m}^{\prime}-\frac{1}{2} b_{m}^{2}-\mathcal{P}_{4 m}^{\prime} / 4+\mathcal{P}_{4 m}^{2} / 8, \varphi_{3}^{\prime}=\mathcal{F}_{4}^{*} / 2-\mathcal{F}_{2}^{* 2} / 2 \\
\varphi_{4}^{\prime}= & \mathcal{F}_{12}^{*} / 2-\mathcal{F}_{6}^{* 2} / 2, \varphi_{5}^{\prime}=b_{3}^{\prime}-b_{1} b_{2}-\mathcal{P}_{43}^{\prime} / 4+\mathcal{P}_{41} \mathcal{P}_{42} / 4 \\
\varphi_{6}^{\prime}= & \mathcal{F}_{8}^{*}-\mathcal{F}_{2}^{*} \mathcal{F}_{6}^{*} .
\end{aligned}
$$

Expressions for the quantities occurring in $f_{C R}^{(0)}, \varphi_{i}, \varphi_{j}^{\prime}$ are given in $[15,16]$.

Hence, the partial free energy of the $n$-th phase layer $F_{n}$ is written as a power series of deviations of basic arguments from their fixed point values. The linear approximation for $F_{n}$ was used in [14]. In the present paper, as well as in the calculations within the $\rho^{4}$ model $[5,6]$, the quadratics of the deviations are also taken into account. It allows one to compare the results of the calculations for $\rho^{4}$ and $\rho^{6}$ models. Let us note that quadratic terms of the RR do not contribute to the elements of the matrix of the RR linearization in the vicinity of the fixed point, and the eigenvalues $E_{l}$ of this matrix and the critical exponent of the correlation length are the same as within the linear approximation for the RR.

Let us find an explicit dependence of $F_{n}$ on the layer number $n$. Using the solutions of the RR, we get for $h_{n}$ and $\alpha_{n}$ :

$$
\begin{aligned}
h_{n}= & h^{(0)}+c_{1} H_{1}\left(u^{(0)}\right)^{-1 / 2} E_{1}^{n}+c_{2} H_{2}\left(u^{(0)}\right)^{-1} E_{2}^{n}+ \\
& +c_{3} H_{3}\left(u^{(0)}\right)^{-3 / 2} E_{3}^{n}+c_{1} c_{2} H_{4}\left(u^{(0)}\right)^{-3 / 2} E_{1}^{n} E_{2}^{n}+ \\
& +c_{1} c_{2}^{2} H_{5}\left(u^{(0)}\right)^{-5 / 2} E_{1}^{n} E_{2}^{2 n}+c_{2}^{2} H_{6}\left(u^{(0)}\right)^{-2} E_{2}^{2 n}+ \\
& +c_{1}^{2} H_{7}\left(u^{(0)}\right)^{-1} E_{1}^{2 n}+c_{1}^{2} c_{2} H_{8}\left(u^{(0)}\right)^{-2} E_{1}^{2 n} E_{2}^{n}+ \\
& +c_{1}^{2} c_{2}^{2} H_{9}\left(u^{(0)}\right)^{-3} E_{1}^{2 n} E_{2}^{2 n}, \\
\alpha_{n}= & \alpha^{(0)}+c_{1} L_{1}\left(u^{(0)}\right)^{-1 / 2} E_{1}^{n}+c_{2} L_{2}\left(u^{(0)}\right)^{-1} E_{2}^{n}+ \\
& +c_{3} L_{3}\left(u^{(0)}\right)^{-3 / 2} E_{3}^{n}+c_{1} c_{2} L_{4}\left(u^{(0)}\right)^{-3 / 2} E_{1}^{n} E_{2}^{n}+ \\
& +c_{1} c_{2}^{2} L_{5}\left(u^{(0)}\right)^{-5 / 2} E_{1}^{n} E_{2}^{2 n}+c_{2}^{2} L_{6}\left(u^{(0)}\right)^{-2} E_{2}^{2 n}+ \\
& +c_{1}^{2} L_{7}\left(u^{(0)}\right)^{-1} E_{1}^{2 n}+c_{1}^{2} c_{2} L_{8}\left(u^{(0)}\right)^{-2} E_{1}^{2 n} E_{2}^{n}+ \\
& +c_{1}^{2} c_{2}^{2} L_{9}\left(u^{(0)}\right)^{-3} E_{1}^{2 n} E_{2}^{2 n},
\end{aligned}
$$


where

$$
\begin{aligned}
& H_{1}=\sqrt{6}-\frac{h^{(0)} w_{21}^{(0)}}{2}, \quad H_{2}=\sqrt{6} w_{12}^{(0)}-\frac{h^{(0)}}{2}, \\
& H_{3}=\sqrt{6} w_{13}^{(0)}-\frac{h^{(0)} w_{23}^{(0)}}{2}, \\
& H_{4}=\frac{3}{4} h^{(0)} w_{21}^{(0)}-\frac{\sqrt{6}}{2}\left(1+w_{12}^{(0)} w_{21}^{(0)}\right), \\
& H_{5}=\frac{3 \sqrt{6}}{4}\left(\frac{1}{2}+w_{12}^{(0)} w_{21}^{(0)}-\frac{5}{4 \sqrt{6}} h^{(0)} w_{21}^{(0)}\right), \\
& H_{6}=\frac{1}{2}\left(\frac{3}{4} h^{(0)}-\sqrt{6} w_{12}^{(0)}\right), \quad H_{7}=\frac{w_{21}^{(0)}}{2}\left(\frac{3}{4} h^{(0)} w_{21}^{(0)}-\sqrt{6}\right), \\
& H_{8}=\frac{3 \sqrt{6}}{4} w_{21}^{(0)}\left(1+\frac{1}{2} w_{12}^{(0)} w_{21}^{(0)}-\frac{5}{4 \sqrt{6}} h^{(0)} w_{21}^{(0)}\right), \\
& H_{9}=\frac{15 \sqrt{6}}{16} w_{21}^{(0)}\left(\frac{7}{4 \sqrt{6}} h^{(0)} w_{21}^{(0)}-1-w_{12}^{(0)} w_{21}^{(0)}\right) ; \\
& L_{1}=\frac{\sqrt{6}}{15} w_{31}^{(0)}-\frac{3 \alpha^{(0)} w_{21}^{(0)}}{2}, \quad L_{2}=\frac{\sqrt{6}}{15} w_{32}^{(0)}-\frac{3 \alpha^{(0)}}{2}, \\
& L_{3}=\frac{\sqrt{6}}{15}-\frac{3 \alpha^{(0)} w_{23}^{(0)}}{2} \text {, } \\
& L_{4}=\frac{15}{4} \alpha^{(0)} w_{21}^{(0)}-\frac{\sqrt{6}}{10}\left(w_{31}^{(0)}+w_{21}^{(0)} w_{32}^{(0)}\right), \\
& L_{5}=\frac{\sqrt{6}}{4}\left(\frac{1}{2} w_{31}^{(0)}+w_{21}^{(0)} w_{32}^{(0)}-\frac{105}{4 \sqrt{6}} \alpha^{(0)} w_{21}^{(0)}\right), \\
& L_{6}=\frac{1}{2}\left(\frac{15}{4} \alpha^{(0)}-\frac{\sqrt{6}}{5} w_{32}^{(0)}\right), \\
& L_{7}=\frac{w_{21}^{(0)}}{2}\left(\frac{15}{4} \alpha^{(0)} w_{21}^{(0)}-\frac{\sqrt{6}}{5} w_{31}^{(0)}\right) \text {, } \\
& L_{8}=\frac{\sqrt{6}}{4} w_{21}^{(0)}\left(w_{31}^{(0)}+\frac{1}{2} w_{21}^{(0)} w_{32}^{(0)}-\frac{105}{4 \sqrt{6}} \alpha^{(0)} w_{21}^{(0)}\right) \text {, } \\
& L_{9}=\frac{7 \sqrt{6}}{16} w_{21}^{(0)}\left(\frac{45}{4 \sqrt{6}} \alpha^{(0)} w_{21}^{(0)}-w_{31}^{(0)}-w_{21}^{(0)} w_{32}^{(0)}\right) \text {. }
\end{aligned}
$$

Considering (2.4), we rewrite the partial energy of the $n$-th phase layer as

$$
\begin{aligned}
F_{n}= & -k T N^{\prime} s^{-3 n}\left[f_{C R}^{(0)}+f_{C R}^{(1)}\left(u^{(0)}\right)^{-1 / 2} c_{1} E_{1}^{n}+f_{C R}^{(2)}\left(u^{(0)}\right)^{-1} c_{2} E_{2}^{n}+\right. \\
& +f_{C R}^{(3)}\left(u^{(0)}\right)^{-3 / 2} c_{3} E_{3}^{n}+f_{C R}^{(4)}\left(u^{(0)}\right)^{-3 / 2} c_{1} c_{2} E_{1}^{n} E_{2}^{n}+ \\
& +f_{C R}^{(5)}\left(u^{(0)}\right)^{-5 / 2} c_{1} c_{2}^{2} E_{1}^{n} E_{2}^{2 n}+f_{C R}^{(6)}\left(u^{(0)}\right)^{-2} c_{2}^{2} E_{2}^{2 n}+ \\
& +f_{C R}^{(7)}\left(u^{(0)}\right)^{-1} c_{1}^{2} E_{1}^{2 n}+f_{C R}^{(8)}\left(u^{(0)}\right)^{-2} c_{1}^{2} c_{2} E_{1}^{2 n} E_{2}^{n}+ \\
& \left.+f_{C R}^{(9)}\left(u^{(0)}\right)^{-3} c_{1}^{2} c_{2}^{2} E_{1}^{2 n} E_{2}^{2 n}\right] .
\end{aligned}
$$


Here,

$$
\begin{aligned}
f_{C R}^{(m)}= & H_{m}\left(\varphi_{3}+\varphi_{1} / E_{m}\right)+L_{m}\left(\varphi_{4}+\varphi_{2} / E_{m}\right), m=1,2,3 \\
f_{C R}^{(4)}= & H_{4}\left(\varphi_{3}+\varphi_{1} /\left(E_{1} E_{2}\right)\right)+L_{4}\left(\varphi_{4}+\varphi_{2} /\left(E_{1} E_{2}\right)\right)+ \\
& +2 H_{1} H_{2}\left(\varphi_{3}^{\prime}+\varphi_{1}^{\prime} /\left(E_{1} E_{2}\right)\right)+2 L_{1} L_{2}\left(\varphi_{4}^{\prime}+\varphi_{2}^{\prime} /\left(E_{1} E_{2}\right)\right)+ \\
& +\left(H_{1} L_{2}+H_{2} L_{1}\right)\left(\varphi_{6}^{\prime}+\varphi_{5}^{\prime} /\left(E_{1} E_{2}\right)\right), \\
f_{C R}^{(5)}= & H_{5}\left(\varphi_{3}+\varphi_{1} /\left(E_{1} E_{2}^{2}\right)\right)+L_{5}\left(\varphi_{4}+\varphi_{2} /\left(E_{1} E_{2}^{2}\right)\right)+ \\
& +2\left(H_{1} H_{6}+H_{2} H_{4}\right)\left(\varphi_{3}^{\prime}+\varphi_{1}^{\prime} /\left(E_{1} E_{2}^{2}\right)\right)+2\left(L_{1} L_{6}+L_{2} L_{4}\right) \times \\
& \times\left(\varphi_{4}^{\prime}+\varphi_{2}^{\prime} /\left(E_{1} E_{2}^{2}\right)\right)+\left(H_{1} L_{6}+H_{6} L_{1}+H_{2} L_{4}+H_{4} L_{2}\right) \times \\
& \times\left(\varphi_{6}^{\prime}+\varphi_{5}^{\prime} /\left(E_{1} E_{2}^{2}\right)\right) \\
& H_{6}\left(\varphi_{3}+\varphi_{1} / E_{2}^{2}\right)+L_{6}\left(\varphi_{4}+\varphi_{2} / E_{2}^{2}\right)+H_{2}^{2}\left(\varphi_{3}^{\prime}+\varphi_{1}^{\prime} / E_{2}^{2}\right)+ \\
& +L_{2}^{2}\left(\varphi_{4}^{\prime}+\varphi_{2}^{\prime} / E_{2}^{2}\right)+H_{2} L_{2}\left(\varphi_{6}^{\prime}+\varphi_{5}^{\prime} / E_{2}^{2}\right), \\
f_{C R}^{(6)}= & H_{7}\left(\varphi_{3}+\varphi_{1} / E_{1}^{2}\right)+L_{7}\left(\varphi_{4}+\varphi_{2} / E_{1}^{2}\right)+H_{1}^{2}\left(\varphi_{3}^{\prime}+\varphi_{1}^{\prime} / E_{1}^{2}\right)+ \\
& +L_{1}^{2}\left(\varphi_{4}^{\prime}+\varphi_{2}^{\prime} / E_{1}^{2}\right)+H_{1} L_{1}\left(\varphi_{6}^{\prime}+\varphi_{5}^{\prime} / E_{1}^{2}\right), \\
f_{C R}^{(7)}= & H_{8}\left(\varphi_{3}+\varphi_{1} /\left(E_{1}^{2} E_{2}\right)\right)+L_{8}\left(\varphi_{4}+\varphi_{2} /\left(E_{1}^{2} E_{2}\right)\right)+2\left(H_{1} H_{4}+\right. \\
& \left.+H_{2} H_{7}\right)\left(\varphi_{3}^{\prime}+\varphi_{1}^{\prime} /\left(E_{1}^{2} E_{2}\right)\right)+2\left(L_{1} L_{4}+L_{2} L_{7}\right)\left(\varphi_{4}^{\prime}+\right. \\
& \left.+\varphi_{2}^{\prime} /\left(E_{1}^{2} E_{2}\right)\right)+\left(H_{1} L_{4}+H_{4} L_{1}+H_{2} L_{7}+H_{7} L_{2}\right) \times \\
& \times\left(\varphi_{6}^{\prime}+\varphi_{5}^{\prime} /\left(E_{1}^{2} E_{2}\right)\right), \\
f_{C R}^{(8)}= & H_{9}\left(\varphi_{3}+\varphi_{1} /\left(E_{1} E_{2}\right)^{2}\right)+L_{9}\left(\varphi_{4}+\varphi_{2} /\left(E_{1} E_{2}\right)^{2}\right)+\left(2 H_{1} H_{5}+\right. \\
& \left.+2 H_{2} H_{8}+H_{4}^{2}+2 H_{6} H_{7}\right)\left(\varphi_{3}^{\prime}+\varphi_{1}^{\prime} /\left(E_{1} E_{2}\right)^{2}\right)+\left(2 L_{1} L_{5}+\right. \\
& \left.+2 L_{2} L_{8}+L_{4}^{2}+2 L_{6} L_{7}\right)\left(\varphi_{4}^{\prime}+\varphi_{2}^{\prime} /\left(E_{1} E_{2}\right)^{2}\right)+\left(H_{1} L_{5}+\right. \\
& \left.+H_{5} L_{1}+H_{2} L_{8}+H_{8} L_{2}+H_{4} L_{4}+H_{6} L_{7}+H_{7} L_{6}\right) \times \\
& \times\left(\varphi_{6}^{\prime}+\varphi_{5}^{\prime} /\left(E_{1} E_{2}\right)^{2}\right) . \\
f_{C R}^{(9)}= &
\end{aligned}
$$

The quantities $u^{(0)}, w_{i l}^{(0)}$ were determined in [13]. Let us note that in the expressions for $h_{n}$ and $\alpha_{n}$ we can neglect a qualitatively new term proportional to $E_{3}^{n}$ which arises within the $\rho^{6}$ model considered ( $E_{3}$ is not essential as compared to $E_{1}$ or $E_{2}$ ).

Summing up expressions for $F_{n}(2.6)$ over layers of the phase space from $n=0$ to $n=m_{\tau}$, we obtain

$$
\begin{aligned}
F_{C R} & =F_{0}^{\prime}+F_{C R}^{\prime}, \\
F_{0}^{\prime} & =-k T N^{\prime}[\ln Q(\mathcal{M})+\ln Q(d)], \\
F_{C R}^{\prime} & =F_{C R}^{(0)}+F_{C R}^{(1)} \tau^{3 \nu}+F_{C R}^{(2)} \tau^{3 \nu},
\end{aligned}
$$

where

$$
\begin{aligned}
F_{C R}^{(0)}= & -k T N^{\prime} s^{-3}\left[\frac{f_{C R}^{(0)}}{1-s^{-3}}+\frac{f_{C R}^{(1)} \varphi_{0}^{-1 / 2} \tilde{c}_{1} \tau E_{1}}{1-E_{1} s^{-3}}+\right. \\
& +\frac{f_{C R}^{(2)} \varphi_{0}^{-1} c_{20} E_{2}}{1-E_{2} s^{-3}}+\frac{f_{C R}^{(3)} \varphi_{0}^{-3 / 2} c_{30} E_{3}}{1-E_{3} s^{-3}}+\frac{f_{C R}^{(4)} \varphi_{0}^{-3 / 2} \tilde{c}_{1} \tau c_{20} E_{1} E_{2}}{1-E_{1} E_{2} s^{-3}}+
\end{aligned}
$$




$$
\begin{aligned}
& +\frac{f_{C R}^{(5)} \varphi_{0}^{-5 / 2} \tilde{c}_{1} \tau c_{20}^{2} E_{1} E_{2}^{2}}{1-E_{1} E_{2}^{2} s^{-3}}+\frac{f_{C R}^{(6)} \varphi_{0}^{-2} c_{20}^{2} E_{2}^{2}}{1-E_{2}^{2} s^{-3}}+ \\
& +\frac{f_{C R}^{(7)} \varphi_{0}^{-1} \tilde{c}_{1}^{2} \tau^{2} E_{1}^{2}}{1-E_{1}^{2} s^{-3}}+\frac{f_{C R}^{(8)} \varphi_{0}^{-2} \tilde{c}_{1}^{2} \tau^{2} c_{20} E_{1}^{2} E_{2}}{1-E_{1}^{2} E_{2} s^{-3}}+ \\
& \left.+\frac{f_{C R}^{(9)} \varphi_{0}^{-3} \tilde{c}_{1}^{2} \tau^{2} c_{20}^{2} E_{1}^{2} E_{2}^{2}}{1-E_{1}^{2} E_{2}^{2} s^{-3}}\right], \\
F_{C R}^{(1)}= & k T N^{\prime} s^{-3 m_{0}}\left[\frac{f_{C R}^{(0)}}{1-s^{-3}}+\frac{f_{C R}^{(1)} \varphi_{0}^{-1 / 2} f_{0}}{1-E_{1} s^{-3}}+\right. \\
& \left.+\frac{f_{C R}^{(7)} \varphi_{0}^{-1} f_{0}^{2}}{1-E_{1}^{2} s^{-3}}\right], \\
F_{C R}^{(2)}= & -k T N^{\prime} s^{-3 m_{0}}\left[\frac{f_{C R}^{(2)} \varphi_{0}^{-1} c_{20} E_{2}^{m_{\tau}+1}}{1-E_{2} s^{-3}}+\frac{f_{C R}^{(3)} \varphi_{0}^{-3 / 2} c_{30} E_{3}^{m_{\tau}+1}}{1-E_{3} s^{-3}}+\right. \\
& +\frac{f_{C R}^{(4)} \varphi_{0}^{-3 / 2} f_{0} c_{20} E_{2}^{m_{\tau}+1}}{1-E_{1} E_{2} s^{-3}}+\frac{f_{C R}^{(5)} \varphi_{0}^{-5 / 2} f_{0} c_{20}^{2} E_{2}^{2\left(m_{\tau}+1\right)}}{1-E_{1} E_{2}^{2} s^{-3}}+ \\
& +\frac{f_{C R}^{(6)} \varphi_{0}^{-2} c_{20}^{2} E_{2}^{2\left(m_{\tau}+1\right)}}{1-E_{2}^{2} s^{-3}}+\frac{f_{C R}^{(8)} \varphi_{0}^{-2} f_{0}^{2} c_{20} E_{2}^{m_{\tau}+1}}{1-E_{1}^{2} E_{2} s^{-3}}+ \\
& \left.+\frac{f_{C R}^{(9)} \varphi_{0}^{-3} f_{0}^{2} c_{20}^{2} E_{2}^{2\left(m_{\tau}+1\right)}}{1-E_{1}^{2} E_{2}^{2} s^{-3}}\right] .
\end{aligned}
$$

To calculate these expressions, we have used the formulas:

$$
\begin{aligned}
s^{-3\left(m_{\tau}+1\right)} & =\tau^{3 \nu} s^{-3 m_{0}}, \\
c_{1}=\tau \tilde{c}_{1} \beta \tilde{\Phi}(0), \quad c_{2} & =c_{20}(\beta \tilde{\Phi}(0))^{2}, \quad c_{3}=c_{30}(\beta \tilde{\Phi}(0))^{3},
\end{aligned}
$$

where $m_{0}, \tilde{c}_{1}, c_{20}, c_{30}$ and $f_{0}, \varphi_{0}$ were defined in [14]. The dependence of $m_{0}$ on $\mathrm{s}$ for the $\rho^{6}$ model under consideration is plotted in figure 1 (solid curve). Here the dashed curve corresponds to the $\rho^{4}$ model. It is easy to notice that, when $s=s^{*}$ ( $s^{*}$ is the value at which $h_{n}$ turns to zero at the fixed point; $s^{*}=3.5862$ for the $\rho^{4}$ model and $s^{*}=2.7349$ for the $\rho^{6}$ model), the values of $m_{0}$ for $\rho^{4}$ and $\rho^{6}$ models coincide.

Further, we put $F_{C R}^{(2)}=0$ at $\tau \ll 1$, since $E_{2}<1, E_{3}<1$, and $m_{\tau}$ is large. Taking $E_{2}, E_{3}$ into account gives rise to terms characterising corrections to scaling. As a result, the free energy of the $\mathrm{CR}$ region takes the form:

$$
\begin{aligned}
F_{C R}= & -k T N^{\prime}\left[\gamma_{0}+\delta_{0}-\gamma_{3}^{(C R)+} \tau^{3 \nu}\right] \\
\gamma_{0}= & s^{-3}\left[\frac{f_{C R}^{(0)}}{1-s^{-3}}+\frac{f_{C R}^{(1)} \varphi_{0}^{-1 / 2} \tilde{c}_{1} \tau E_{1}}{1-E_{1} s^{-3}}+\frac{f_{C R}^{(2)} \varphi_{0}^{-1 / 2} c_{20} E_{2}}{1-E_{2} s^{-3}}+\right. \\
& +\frac{f_{C R}^{(3)} \varphi_{0}^{-3 / 2} c_{30} E_{3}}{1-E_{3} s^{-3}}+\frac{f_{C R}^{(4)} \varphi_{0}^{-3 / 2} \tilde{c}_{1} \tau c_{20} E_{1} E_{2}}{1-E_{1} E_{2} s^{-3}}+ \\
& +\frac{f_{C R}^{(5)} \varphi_{0}^{-5 / 2} \tilde{c}_{1} c_{20}^{2} \tau E_{1} E_{2}^{2}}{1-E_{1} E_{2}^{2} s^{-3}}+\frac{f_{C R}^{(6)} \varphi_{0}^{-2} c_{20}^{2} E_{2}^{2}}{1-E_{2}^{2} s^{-3}}+\frac{f_{C R}^{(7)} \varphi_{0}^{-1} \tilde{c}_{1}^{2} \tau^{2} E_{1}^{2}}{1-E_{1}^{2} s^{-3}}+ \\
& \left.+\frac{f_{C R}^{(8)} \varphi_{0}^{-2} \tilde{c}_{1}^{2} c_{20} \tau^{2} E_{1}^{2} E_{2}}{1-E_{1}^{2} E_{2} s^{-3}}+\frac{f_{C R}^{(9)} \varphi_{0}^{-3} \tilde{c}_{1}^{2} c_{20}^{2} \tau^{2} E_{1}^{2} E_{2}^{2}}{1-E_{1}^{2} E_{2}^{2} s^{-3}}\right],
\end{aligned}
$$




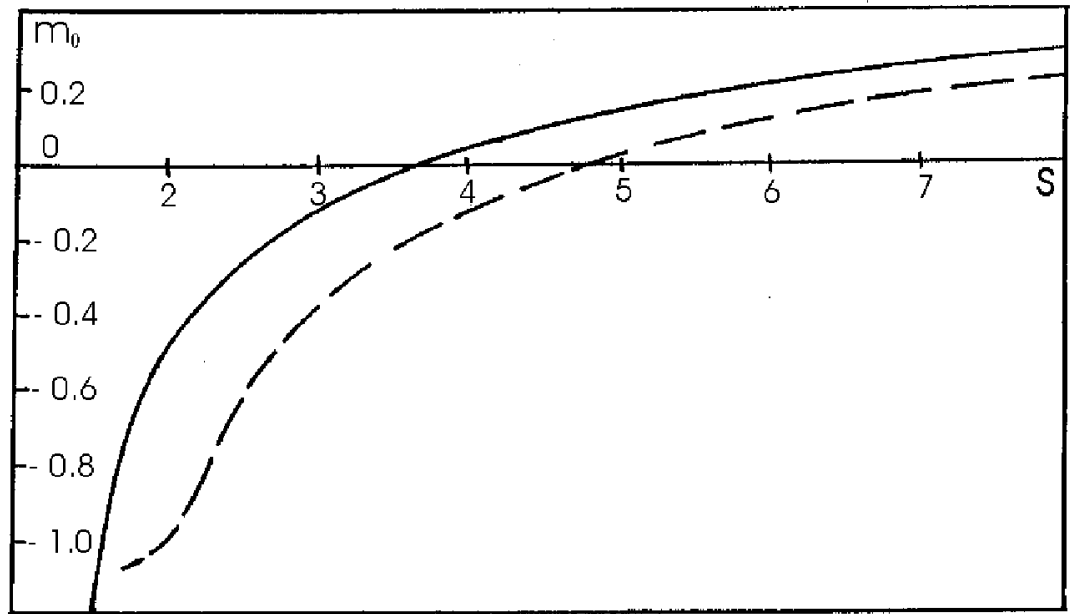

Figure 1. Dependence of the quantity $m_{0}$ on the RG parameter $s$ for the $\rho^{6}$ model (solid curve) and the $\rho^{4}$ model (dashed curve).

$$
\begin{aligned}
\delta_{0} & =\ln Q(\mathcal{M})+\ln Q(d), \quad \gamma_{3}^{(C R)+}=c_{\nu}^{3} \gamma^{+}, \quad c_{\nu}=\left(\frac{\tilde{c}_{1}^{(0)}}{f_{0}}\right)^{\nu}, \\
\gamma^{+} & =\frac{f_{C R}^{(0)}}{1-s^{-3}}+\frac{f_{C R}^{(1)} \varphi_{0}^{-1 / 2} f_{0}}{1-E_{1} s^{-3}}+\frac{f_{C R}^{(7)} \varphi_{0}^{-1} f_{0}^{2}}{1-E_{1}^{2} s^{-3}} .
\end{aligned}
$$

Note that $\gamma_{0}, \delta_{0}$ are the functions of temperature, since they are expressed in terms of $\tilde{c}_{1}, c_{20}, c_{30}$ and $Q(d)$. Let us extract the temperature dependence in these quantities.

Near $T_{c}$ we have for $\tilde{c}_{1}$

$$
\begin{aligned}
\tilde{c}_{1} & =\tilde{c}_{1}^{(0)}+\tilde{c}_{1}^{(1)} \tau, \\
\tilde{c}_{1}^{(0)} & =V_{1}\left[1-f_{0}+v_{12}^{(0)} \varphi_{0}^{1 / 2}+v_{13}^{(0)} \psi_{0} \varphi_{0}^{-1}+\frac{a_{4}^{\prime} v_{12}^{(0)} \varphi_{0}^{-1 / 2}}{\left(\beta_{c} \tilde{\Phi}(0)\right)^{2}}+\right. \\
& \left.+\frac{2 a_{6}^{\prime} v_{13}^{(0)} \varphi_{0}^{-1}}{\left(\beta_{c} \tilde{\Phi}(0)\right)^{3}}\right], \\
\tilde{c}_{1}^{(1)} & =V_{1}\left[\frac{a_{4}^{\prime} v_{12}^{(0)} \varphi_{0}^{-1 / 2}}{\left(\beta_{c} \tilde{\Phi}(0)\right)^{2}}+\frac{3 a_{6}^{\prime} v_{13}^{(0)} \varphi_{0}^{-1}}{\left(\beta_{c} \tilde{\Phi}(0)\right)^{3}}\right],
\end{aligned}
$$

for $c_{20}$ and $c_{30}$ we get, respectively,

$$
\begin{aligned}
c_{20} & =c_{20}^{(0)}+c_{20}^{(1)} \tau+c_{20}^{(2)} \tau^{2}, \\
c_{20}^{(0)} & =V_{2}\left[-\varphi_{0}-v_{21}^{(0)}\left(1-f_{0}\right) \varphi_{0}^{1 / 2}-v_{23}^{(0)} \psi_{0} \varphi_{0}^{-1 / 2}+\frac{a_{2}^{\prime} v_{21}^{(0)} \varphi_{0}^{1 / 2}}{\beta_{c} \tilde{\Phi}(0)}+\right.
\end{aligned}
$$




$$
\begin{aligned}
+ & \left.\frac{a_{4}^{\prime}}{\left(\beta_{c} \tilde{\Phi}(0)\right)^{2}}+\frac{a_{6}^{\prime} v_{23}^{(0)} \varphi_{0}^{-1 / 2}}{\left(\beta_{c} \tilde{\Phi}(0)\right)^{3}}\right], \\
c_{20}^{(1)}= & V_{2}\left[\frac{a_{2}^{\prime} v_{21}^{(0)} \varphi_{0}^{1 / 2}}{\beta_{c} \tilde{\Phi}(0)}+\frac{2 a_{4}^{\prime}}{\left(\beta_{c} \tilde{\Phi}(0)\right)^{2}}+\frac{3 a_{6}^{\prime} v_{23}^{(0)} \varphi_{0}^{-1 / 2}}{\left(\beta_{c} \tilde{\Phi}(0)\right)^{3}}\right], \\
c_{20}^{(2)}= & V_{2}\left[\frac{a_{4}^{\prime}}{\left(\beta_{c} \tilde{\Phi}(0)\right)^{2}}+\frac{3 a_{6}^{\prime} v_{23}^{(0)} \varphi_{0}^{-1 / 2}}{\left(\beta_{c} \tilde{\Phi}(0)\right)^{3}}\right] ; \\
c_{30}= & c_{30}^{(0)}+c_{30}^{(1)} \tau+c_{30}^{(2)} \tau^{2}, \\
c_{30}^{(0)}= & V_{3}\left[-\psi_{0}-\varphi_{0}^{3 / 2} v_{32}^{(0)}-\varphi_{0}\left(1-f_{0}\right) v_{31}^{(0)}+\frac{a_{2}^{\prime} v_{31}^{(0)} \varphi_{0}}{\beta_{c} \tilde{\Phi}(0)}+\right. \\
& \left.+\frac{a_{4}^{\prime} v_{32}^{(0)} \varphi_{0}^{1 / 2}}{\left(\beta_{c} \tilde{\Phi}(0)\right)^{2}}+\frac{a_{6}^{\prime}}{\left(\beta_{c} \tilde{\Phi}(0)\right)^{3}}\right], \\
c_{30}^{(1)}= & V_{3}\left[\frac{a_{2}^{\prime} v_{31}^{(0)} \varphi_{0}}{\beta_{c} \tilde{\Phi}(0)}+\frac{2 a_{4}^{\prime} v_{32}^{(0)} \varphi_{0}^{1 / 3}}{\left(\beta_{c} \tilde{\Phi}(0)\right)^{2}}+\frac{3 a_{6}^{\prime}}{\left(\beta_{c} \tilde{\Phi}(0)\right)^{3}}\right], \\
c_{30}^{(2)}= & V_{3}\left[\frac{a_{4}^{\prime} v_{32}^{(0)} \varphi_{0}^{1 / 2}}{\left(\beta_{c} \tilde{\Phi}(0)\right)^{2}}+\frac{3 a_{6}^{\prime}}{\left(\beta_{c} \tilde{\Phi}(0)\right)^{3}}\right] .
\end{aligned}
$$

The values of $\beta_{c} \tilde{\Phi}(0)$, the correlation length critical exponent $\nu=\ln s / \ln E_{1}$, exponents of the corrections to scaling $\Delta_{1}=-\ln E_{2} / \ln E_{1}, \Delta_{2}=$ $-\ln E_{3} / \ln E_{1}$, and coefficients of the expressions for $\tilde{c}_{1}, c_{20}, c_{30}$ are given in tables 1,2 . In the present paper, the numerical calculations are performed at $b / c=1$ and arithmetically averaged Fourier transform of the potential.

Table 1. Values of $\beta_{c} \tilde{\Phi}(0), \nu, \Delta_{1}, \Delta_{2}, \tilde{c}_{1}^{(0)}, \tilde{c}_{1}^{(1)}$ for different $s$.

\begin{tabular}{lllllll}
\hline$s$ & $\beta_{c} \tilde{\Phi}(0)$ & $\nu$ & $\Delta_{1}$ & $\Delta_{2}$ & $\tilde{c}_{1}^{(0)}$ & $\tilde{c}_{1}^{(1)}$ \\
\hline 2 & 1.1204 & 0.619 & 0.653 & 5.061 & 0.7602 & 0.0109 \\
2.5 & 1.1405 & 0.634 & 0.552 & 3.963 & 0.7637 & 0.0103 \\
2.7349 & 1.1506 & 0.637 & 0.525 & 3.647 & 0.7641 & 0.0100 \\
3 & 1.1628 & 0.640 & 0.503 & 3.379 & 0.7630 & 0.0096 \\
3.5 & 1.1882 & 0.645 & 0.476 & 3.038 & 0.7570 & 0.0089 \\
3.5862 & 1.1929 & 0.645 & 0.473 & 2.994 & 0.7555 & 0.0088 \\
4 & 1.2165 & 0.648 & 0.460 & 2.821 & 0.7471 & 0.0082 \\
& & & & & & \\
\hline
\end{tabular}

Expressions for the quantities $V_{1}, V_{2}, V_{3}, \psi_{0}, v_{l j}^{(0)}$ occurring in (2.12) (2.14) are presented in $[13,14]$. The coefficient $\gamma_{0}$ can be written as

$$
\begin{aligned}
\gamma_{0} & =\gamma_{0}^{(0)}+\gamma_{0}^{(1)} \tau+\gamma_{0}^{(2)} \tau^{2}, \\
\gamma_{0}^{(0)} & =s^{-3}\left[\frac{f_{C R}^{(0)}}{1-s^{-3}}+\frac{f_{C R}^{(2)} \varphi_{0}^{-1} c_{20}^{(0)} E_{2}}{1-E_{2} s^{-3}}+\frac{f_{C R}^{(3)} \varphi_{0}^{-3 / 2} c_{30}^{(0)} E_{3}}{1-E_{3} s^{-3}}+\right.
\end{aligned}
$$


Table 2. Coefficients in equations for $c_{20}(2.13)$ and $c_{30}(2.14)$.

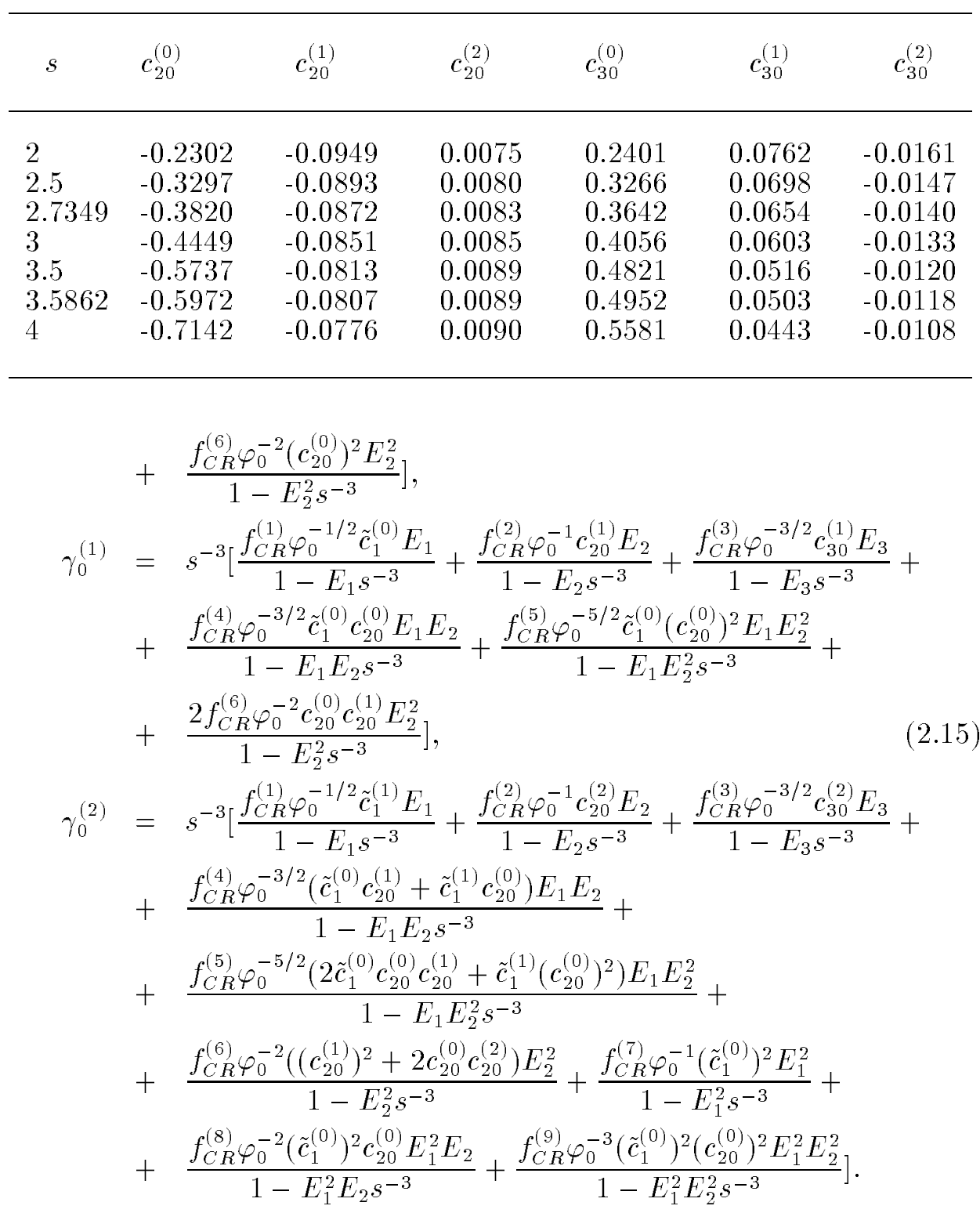

For $\delta_{0}$ we obtain

$$
\begin{aligned}
\delta_{0} & =\delta_{0}^{(0)}+\delta_{0}^{(1)} \tau+\delta_{0}^{(2)} \tau^{2} \\
\delta_{0}^{(0)} & =\ln Q(\mathcal{M})+\ln Q\left(d, T_{c}\right), \\
\delta_{0}^{(1)} & =-\frac{\sqrt{6}}{\sqrt{a_{4}^{\prime}}}(1-\bar{q}) \beta_{c} \tilde{\Phi}(0) \mathcal{F}_{2}\left(h_{c}, \alpha\right), \\
\delta_{0}^{(2)} & =-\frac{3}{a_{4}^{\prime}}(1-\bar{q})^{2}\left(\beta_{c} \tilde{\Phi}(0)\right)^{2}\left[\mathcal{F}_{2}^{2}\left(h_{c}, \alpha\right)-\mathcal{F}_{4}\left(h_{c}, \alpha\right)\right]+
\end{aligned}
$$




$$
\begin{aligned}
& +\frac{\sqrt{6}}{\sqrt{a_{4}^{\prime}}}(1-\bar{q}) \beta_{c} \tilde{\Phi}(0) \mathcal{F}_{2}\left(h_{c}, \alpha\right), \\
h_{c} & =\frac{\sqrt{6}}{\sqrt{a_{4}^{\prime}}}\left[a_{2}^{\prime}-\beta_{c} \tilde{\Phi}(0)(1-\bar{q})\right], \quad \alpha=\frac{\sqrt{6}}{15} \frac{a_{6}^{\prime}}{\left(a_{4}^{\prime}\right)^{3 / 2}}, \quad \bar{q}=\frac{1+s^{-2}}{2} .
\end{aligned}
$$

Coefficients of the expressions for $\gamma_{0}(2.15), \delta_{0}(2.16)$ at different values of the RG parameter $s$ are given in table 3 .

Hence, the free energy of the $\mathrm{CR}$ region reads

$$
\begin{aligned}
F_{C R} & =-k T N^{\prime}\left[\gamma_{0}^{(C R)}+\gamma_{1} \tau+\gamma_{2} \tau^{2}+\gamma_{3}^{(C R)+} \tau^{3 \nu}\right], \\
\gamma_{0}^{(C R)} & =\gamma_{0}^{(0)}+\delta_{0}^{(0)}, \\
\gamma_{1} & =\gamma_{0}^{(1)}+\delta_{0}^{(1)}, \\
\gamma_{2} & =\gamma_{0}^{(2)}+\delta_{0}^{(2)} .
\end{aligned}
$$

The numerical values of coefficients $\gamma_{0}^{(C R)}, \gamma_{1}, \gamma_{2}$ and quantities $\gamma^{+}, c_{\nu}=$ $\left(\tilde{c}_{1}^{(0)} / f_{0}\right)^{\nu}$ occurring in $\gamma_{3}^{(C R)+}$ are given in table 4 .

Knowledge of $F_{C R}$ allows one to calculate other thermodynamic functions of the system in the $\mathrm{CR}$ region at $T>T_{c}$. For the entropy $S_{C R}$, internal energy $U_{C R}$ and specific heat $C_{C R}$ we get

$$
\begin{aligned}
S_{C R} & =k N^{\prime}\left[s^{(0)(C R)}+c_{0} \tau+u_{3}^{(C R)+} \tau^{1-\alpha}\right], \\
U_{C R} & =k T N^{\prime}\left[\gamma_{1}+u_{1} \tau+u_{3}^{(C R)+} \tau^{1-\alpha}\right], \\
C_{C R} & =k N^{\prime}\left[c_{0}+c_{3}^{(C R)+} \tau^{-\alpha}\right], \\
s^{(0)(C R)} & =\gamma_{0}^{(C R)}+\gamma_{1}, \quad c_{0}=2\left(\gamma_{1}+\gamma_{2}\right), \quad u_{3}^{(C R)+}=-3 \nu \gamma_{3}^{(C R)+}, \\
u_{1} & =2 \gamma_{2}+\gamma_{1}, \quad c_{3}^{(C R)+}=-3 \nu(3 \nu-1) \gamma_{3}^{(C R)+}, \quad \alpha=2-3 \nu .
\end{aligned}
$$

In the region of LGR, the expression for the part $Z_{L G R}$ of the partition function (2.1) reads

$$
Z_{L G R}=\int \exp \left\{-\frac{1}{2} \sum_{k \leqslant B_{m_{\tau}+1}}\left[d_{m_{\tau}}(k)-d_{m_{\tau}}\left(B_{m_{\tau+1}}, B_{m_{\tau}}\right)\right] \rho_{\boldsymbol{k}} \rho_{-\boldsymbol{k}}-\right.
$$

Table 3. Values of coefficients in equations for $\gamma_{0}(2.15)$ and $\delta_{0}(2.16)$.

\begin{tabular}{lllllll}
\hline \multicolumn{1}{c}{$s$} & $\gamma_{0}^{(0)}$ & $\gamma_{0}^{(1)}$ & $\gamma_{0}^{(2)}$ & $\delta_{0}^{(0)}$ & $\delta_{0}^{(1)}$ & $\delta_{0}^{(2)}$ \\
\hline 2 & 0.1205 & -0.3160 & -2.5291 & 0.2711 & -0.3574 & 0.4810 \\
2.5 & 0.0757 & -0.2343 & -2.9320 & 0.3237 & -0.4510 & 0.6464 \\
2.7349 & 0.0624 & -0.2062 & -2.9801 & 0.3423 & -0.4862 & 0.7128 \\
3 & 0.0510 & -0.1798 & -2.9798 & 0.3606 & -0.5222 & 0.7827 \\
3.5 & 0.0362 & -0.1415 & -2.9314 & 0.3905 & -0.5836 & 0.9075 \\
3.5862 & 0.0343 & -0.1360 & -2.9236 & 0.3953 & -0.5938 & 0.9287 \\
4 & 0.0267 & -0.1135 & -2.9039 & 0.4175 & -0.6420 & 1.0319 \\
& & & & & & \\
\hline
\end{tabular}


Table 4. Coefficients $\gamma_{0}^{(C R)}, \gamma_{1}, \gamma_{2}$ and quantities $c_{\nu}, \gamma^{+}, \gamma^{-}$contained in $\gamma_{3}^{(C R) \pm}$ for different values of the parameter $s$.

\begin{tabular}{|c|c|c|c|c|c|c|}
\hline$s$ & $\gamma_{0}^{(C R)}$ & $\gamma_{1}$ & $\gamma_{2}$ & $c_{\nu}$ & $\gamma^{+}$ & $\gamma^{-}$ \\
\hline $\begin{array}{l}2 \\
2.5 \\
2.7349 \\
3 \\
3.5 \\
3.5862 \\
4\end{array}$ & $\begin{array}{l}0.3915 \\
0.3994 \\
0.4047 \\
0.4116 \\
0.4267 \\
0.4295 \\
0.4442\end{array}$ & $\begin{array}{l}-0.6734 \\
-0.6852 \\
-0.6924 \\
-0.7020 \\
-0.7251 \\
-0.7298 \\
-0.7555\end{array}$ & $\begin{array}{l}-2.0482 \\
-2.2856 \\
-2.2672 \\
-2.1971 \\
-2.0239 \\
-1.9949 \\
-1.8720\end{array}$ & $\begin{array}{l}1.4412 \\
1.2722 \\
1.2097 \\
1.1462 \\
1.0414 \\
1.0250 \\
0.9517\end{array}$ & $\begin{array}{l}-0.3170 \\
-0.7757 \\
-0.9831 \\
-1.2229 \\
-1.7450 \\
-1.8496 \\
-2.4345\end{array}$ & $\begin{array}{r}0.7382 \\
0.5244 \\
0.4188 \\
0.2899 \\
-0.0277 \\
-0.0973 \\
-0.5148\end{array}$ \\
\hline & $\begin{array}{l}- \\
- \\
- \\
x\end{array}$ & $\begin{array}{l}\sum_{k \leqslant B_{m_{\tau}+1}} \omega \\
\frac{(2 \pi)^{2 l}}{(2 l) !} N_{m}^{1} \\
\left.+\cdots+\boldsymbol{k}_{2 l}\right\}(d)\end{array}$ & $\begin{array}{c}-\frac{(2 \pi)^{2}}{2} \\
\sum_{k_{1}, \ldots, k_{2 l} \leqslant 1} \\
m_{\tau}+1 \\
(d \omega)\end{array}$ & $\begin{array}{c}\sum_{B_{m_{\tau}+1}} P_{2}^{(} \\
P_{2 l}^{\left(m_{\tau}\right)}\end{array}$ & $\omega_{k} \omega_{-k}$ & (2.19 \\
\hline
\end{tabular}

To calculate $Z_{L G R}$ it is convenient to select two regions of the wave vector values [14]. The first, transition region, corresponds to the values of $k$ close to $B_{m_{\tau}}$; the second, Gaussian region, corresponds to small values of the wave vector $(k \rightarrow 0)$. Hence, we have

$$
Z_{L G R}=Z_{L G R}^{(1)} Z_{L G R}^{(2)}
$$

The contribution to free energy from the phase space layers following the point of exit from the $\mathrm{CR}$ region is

$$
\begin{aligned}
& F_{L G R}^{(1)}=-k T N^{\prime} f_{T R} \tau^{3 \nu}, \\
& f_{T R}=c_{\nu}^{3} \bar{f}_{T R}, \quad \bar{f}_{T R}=\sum_{m=0}^{\tilde{m}_{0}} s^{-3 m} f_{L G R_{1}}(m), \\
& f_{L G R_{1}}(m)=\ln \frac{2}{\pi}+\frac{1}{4} \ln 24-\frac{1}{4} \ln C\left(\eta_{m_{\tau}+m}, \xi_{m_{\tau}+m}\right)+ \\
& +\ln I_{0}\left(h_{m_{\tau}+m+1}, \alpha_{m_{\tau}+m+1}\right)+\ln I_{0}\left(\eta_{m_{\tau}+m}, \xi_{m_{\tau}+m}\right),
\end{aligned}
$$

where $\tilde{m}_{0}$ is the nearest integer to $\tilde{m}_{0}^{\prime}$. The quantities $\tilde{m}_{0}^{\prime}, \eta_{m_{\tau}+m}, \xi_{m_{\tau}+m}$, $h_{m_{\tau}+m+1}, \alpha_{m_{\tau}+m+1}$ were determined in [14]. The plots of $\tilde{m}_{0}^{\prime}(s)$ and analogous dependence $m_{0}{ }^{\prime \prime}(s)$ ( $\rho^{4}$ model) are represented in figure 2 .

Introducing an infinitely small external magnetic field $h=\mu_{B} \mathcal{H}\left(\mu_{B}\right.$ is the Bohr magneton), we can write the part of the free energy corresponding to $Z_{L G R}^{(2)}$ as

$$
F_{L G R}^{(2)}=-k T N^{\prime} f^{\prime} \tau^{3 \nu}-\beta N \gamma_{4}^{+} h^{2} \tau^{-2 \nu}, \quad f^{\prime}=c_{\nu}^{3} \bar{f}^{\prime},
$$




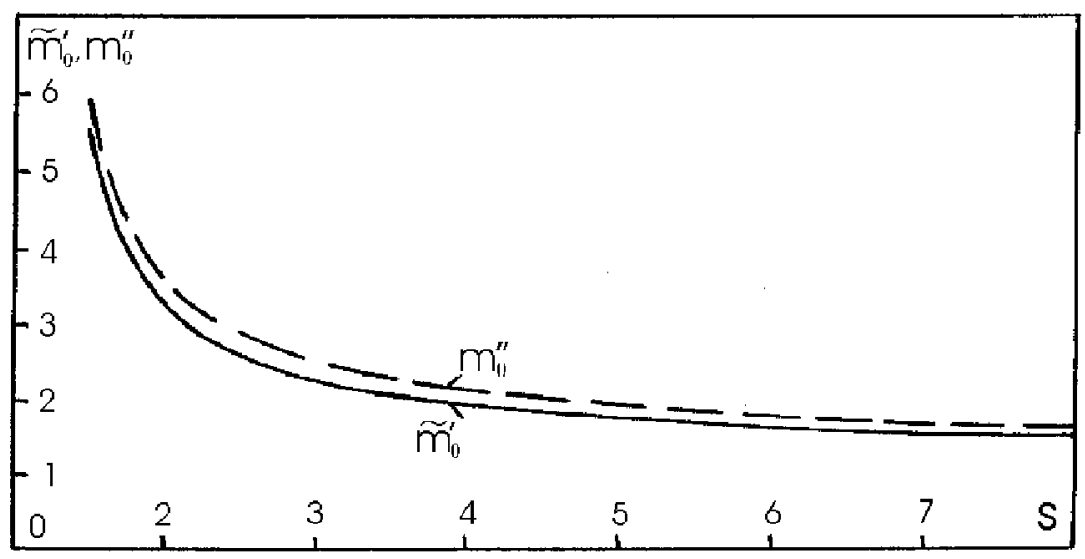

Figure 2. Behaviour of $\tilde{m}_{0}^{\prime}\left(\rho^{6}\right.$ model $)$ and $m_{0}^{\prime \prime}\left(\rho^{4}\right.$ model $)$ with the increase of the parameter $s$.

$$
\begin{aligned}
\bar{f}^{\prime} & =s^{-3\left(\tilde{m}_{0}+1\right)} f_{L G R_{2}}, \\
f_{L G R_{2}} & =-\frac{1}{4} \ln 24+\frac{1}{3}+\frac{1}{4} \ln \bar{u}_{m_{\tau}{ }^{\prime}-1}-\frac{1}{2} \ln \left(\tilde{G}+\frac{1}{s^{2}}\right)- \\
& -\frac{1}{2} \ln \mathcal{F}_{2}\left(h_{m_{\tau}{ }^{\prime}-1}, \alpha_{m_{\tau}{ }^{\prime}-1}\right)-\tilde{G} s^{2}+\left(\tilde{G} s^{2}\right)^{3 / 2} \arctan \left[\left(\tilde{G} s^{2}\right)^{-1 / 2}\right], \\
\gamma_{4}^{+} & =c_{\nu}^{-2} \bar{\gamma}_{4}^{+} /(\beta \tilde{\Phi}(0)), \quad \bar{\gamma}_{4}^{+}=s^{2 \tilde{m}_{0}} /(2 \tilde{G}) .
\end{aligned}
$$

Here,

$$
\begin{aligned}
& m_{\tau}^{\prime}=m_{\tau}+\tilde{m}_{0}+2, \quad \bar{u}_{m_{\tau}^{\prime}-1}=u_{m_{\tau}^{\prime}-1}(\beta \tilde{\Phi}(0))^{-2}, \\
& \tilde{G}=\left(\bar{u}_{m_{\tau}^{\prime}-1} / 24\right)^{1 / 2}\left[\mathcal{F}_{2}\left(h_{m_{\tau}{ }^{\prime}-1}, \alpha_{m_{\tau}-1}\right)\right]^{-1}-\bar{q} .
\end{aligned}
$$

A general expression describing the contribution of long-wave fluctuations to the free energy (LGR region) reads

$$
\begin{aligned}
F_{L G R} & =-k T N^{\prime} f_{L G R} \tau^{3 \nu}-\beta N \gamma_{4}{ }^{+} h^{2} \tau^{-2 \nu}, \\
f_{L G R} & =c_{\nu}^{3} \bar{f}_{L G R}, \quad \bar{f}_{L G R}=\bar{f}_{T R}+\bar{f}^{\prime} .
\end{aligned}
$$

The values of $\bar{f}_{T R}, \quad \bar{f}^{\prime}, \quad \bar{\gamma}_{4}^{+}$are given in table 5 .

The entropy, internal energy, specific heat corresponding to the LGR region are defined by the relations

$$
\begin{aligned}
& S_{L G R}=k N^{\prime} u_{3}^{(L G R)} \tau^{1-\alpha}, \\
& U_{L G R}=k T N^{\prime} u_{3}^{(L G R)} \tau^{1-\alpha}, \\
& C_{L G R}=k N^{\prime} c_{3}^{L G R} \tau^{-\alpha}, \\
& u_{3}^{(L G R)}=3 \nu f_{L G R}, \\
& c_{3}^{(L G R)}=3 \nu(3 \nu-1) f_{L G R} .
\end{aligned}
$$


Table 5. Values of $\bar{f}_{T R}, \bar{f}^{\prime}, \bar{\gamma}_{4}^{+}$.

\begin{tabular}{llll}
\hline \multicolumn{1}{c}{$s$} & $\bar{f}_{T R}$ & $\bar{f}^{\prime} \times 10^{5}$ & $\bar{\gamma}_{4}^{+}$ \\
\hline 2 & & & \\
2.5 & 0.6529 & 0.4749 & 2.7055 \\
2.7349 & 0.8142 & 0.0102 & 3.0870 \\
3 & 0.9541 & 0.2155 & 2.1737 \\
3.5 & 1.0756 & 0.0659 & 2.1841 \\
3.5862 & 1.0950 & 0.0091 & 2.2058 \\
4 & 1.1822 & 0.0067 & 2.2098 \\
& & & 2.2307 \\
\hline
\end{tabular}

\section{Contributions to the thermodynamic functions of the model from the critical and inverse Gaussian regime (CR and IGR) regions below $T_{c}$}

The free energy at $T<T_{c}$ can be written as $[6,10]$

$$
F=F_{0}+F_{C R}+F_{I G R},
$$

where $F_{0}=-k T N \ln 2$ is the free energy of the system of $N$ non-interacting spins, $F_{C R}$ is the contribution to the free energy from the short-wave fluctuation phases of the spin moment density (CR region), and $F_{I G R}$ is the contribution from the long-wave phases of the fluctuations (IGR region).

The number $\mu_{\tau}$ of the $\mathrm{CV}$ phase space layer, separating the short-wave and long-wave phases of fluctuations, is an important characteristic of the system. It is determined from the equation

$$
\frac{r_{\mu_{\tau}+1}-r^{(0)}}{r^{(0)}}=\delta .
$$

Here $r^{(0)}$ corresponds to the fixed point of the $\mathrm{RR}, r_{\mu_{\tau}+1}$ is determined from the solutions of the RR equations (see, for example, $[13,14]$ ), $\delta$ is a constant $(\delta \leqslant 1)$. In the present paper we put $\delta=1$ (see [8]). Let us write an equation for $\mu_{\tau}$

$$
|\tau| \tilde{c}_{1} E_{1}^{\mu_{\tau}+1}=f_{0},
$$

the solution of which is

$$
\mu_{\tau}=-\frac{\ln |\tau|}{\ln E_{1}}+\mu_{0}-1, \quad \mu_{0}=\frac{\ln f_{0}-\ln \tilde{c}_{1}^{(0)}}{\ln E_{1}} .
$$

We need to sum the partial free energies over the layers of the CV phase space to calculate $F_{C R}$. Extracting an explicit dependence on the layer number, using relations (3.3) and

$$
s^{-3\left(\mu_{\tau}+1\right)}=|\tau|^{3 \nu} s^{-3 \mu_{0}}, \quad s^{-3 \mu_{0}}=c_{\nu}^{3},
$$


we obtain

$$
F_{C R}=-k T N^{\prime}\left[\gamma_{0}^{(C R)}-\gamma_{1}|\tau|+\gamma_{2}|\tau|^{2}-\gamma_{3}^{(C R)-}|\tau|^{3 \nu}\right] .
$$

Coefficients $\gamma_{0}^{(C R)}, \gamma_{1}, \gamma_{2}$ are determined in (2.17),

$$
\begin{aligned}
\gamma_{3}^{(C R)-} & =c_{\nu}^{3} \gamma^{-}, \\
\gamma^{-} & =\frac{f_{C R}^{(0)}}{1-s^{-3}}-\frac{f_{C R}^{(1)} \varphi_{0}^{-1 / 2} f_{0}}{1-E_{1} s^{-3}}+\frac{f_{C R}^{(7)} \varphi_{0}^{-1} f_{0}^{2}}{1-E_{1}^{2} s^{-3}}
\end{aligned}
$$

The value of $\gamma^{-}$is given in table 4 .

The entropy, internal energy and specific heat of the system corresponding to the $\mathrm{CR}$ region read

$$
\begin{aligned}
& S_{C R}=k N^{\prime}\left[s^{(0)(C R)}-c_{0}|\tau|+u_{3}^{(C R)-}|\tau|^{1-\alpha}\right], \\
& U_{C R}=k T N^{\prime}\left[\gamma_{1}-u_{1}|\tau|+u_{3}^{(C R)-}|\tau|^{1-\alpha}\right], \\
& C_{C R}=k N^{\prime}\left[c_{0}-c_{3}^{(C R)-}|\tau|^{-\alpha}\right], \\
& u_{3}^{(C R)-}=3 \nu \gamma_{3}^{(C R)-}, \\
& c_{3}^{(C R)-}=3 \nu(3 \nu-1) \gamma_{3}^{(C R)-} .
\end{aligned}
$$

Let us calculate now the contribution to the free energy from the IGR region

$$
F_{I G R}=-k T N^{\prime} s^{-3\left(\mu_{\tau}+1\right)} \ln \left[\sqrt{2} Q\left(P_{\mu_{\tau}}\right)\right]-k T \ln Z_{\mu_{\tau}+1}
$$

where

$$
\begin{aligned}
& \sqrt{2} Q\left(P_{\mu_{\tau}}\right)=\left(\frac{4 s^{3} a_{4}^{\left(\mu_{\tau}\right)}}{\pi^{4} C\left(h_{\mu_{\tau}}, \alpha_{\mu_{\tau}}\right)}\right)^{1 / 4} I_{0}\left(\eta_{\mu_{\tau}}, \xi_{\mu_{\tau}}\right), \\
& Z_{\mu_{\tau}+1}=\int \exp \left\{-\frac{1}{2} \sum_{k \leqslant B_{\mu_{\tau}+1}} d_{\mu_{\tau}+1}(k) \rho_{\boldsymbol{k}} \rho_{-\boldsymbol{k}}-\right. \\
& \left.-\sum_{l=2}^{3} \frac{a_{2 l}^{\left(\mu_{\tau}+1\right)}}{(2 l) !} N_{\mu_{\tau}+1}^{1-l} \sum_{k_{1}, \ldots, k_{2 l} \leqslant B_{\mu_{\tau}+1}} \rho_{\boldsymbol{k}_{1}} \cdots \rho_{\boldsymbol{k}_{2 l}} \delta_{\boldsymbol{k}_{1}+\cdots+\boldsymbol{k}_{2 l}}\right](d \rho)^{N_{\mu_{\tau}+1}} .
\end{aligned}
$$

Consider the first term on the RHS of (3.9). Making use of the relations

$$
\begin{aligned}
r_{\mu_{\tau}} & =-\bar{r}_{\mu_{\tau}} \beta \tilde{\Phi}(0), \quad \bar{r}_{\mu_{\tau}}=f_{0}\left(1+E_{1}^{-1}\right), \\
u_{\mu_{\tau}} & =\bar{u}_{\mu_{\tau}}(\beta \tilde{\Phi}(0))^{2}, \quad \bar{u}_{\mu_{\tau}}=\varphi_{0}-f_{0} \varphi_{0}^{1 / 2} w_{21}^{(0)} E_{1}^{-1}, \\
w_{\mu_{\tau}} & =\bar{w}_{\mu_{\tau}}(\beta \tilde{\Phi}(0))^{3}, \quad \bar{w}_{\mu_{\tau}}=\psi_{0}-f_{0} \varphi_{0} w_{31}^{(0)} E_{1}^{-1},
\end{aligned}
$$

we find

$$
\begin{aligned}
& h_{\mu_{\tau}}=\sqrt{6} \frac{\bar{q}-\bar{r}_{\mu_{\tau}}}{\sqrt{u_{\mu_{\tau}}}}, \quad \alpha_{\mu_{\tau}}=\frac{\sqrt{6}}{15} \frac{\bar{w}_{\mu_{\tau}}}{\left(\bar{u}_{\mu_{\tau}}\right)^{3 / 2}}, \\
& \eta_{\mu_{\tau}}=\sqrt{6} s^{3 / 2} \mathcal{F}_{2}\left(h_{\mu_{\tau}}, \alpha_{\mu_{\tau}}\right)\left[C\left(h_{\mu_{\tau}}, \alpha_{\mu_{\tau}}\right)\right]^{-1 / 2}, \\
& \xi_{\mu_{\tau}}=\frac{\sqrt{6}}{15} s^{-3 / 2} N\left(h_{\mu_{\tau}}, \alpha_{\mu_{\tau}}\right)\left[C\left(h_{\mu_{\tau}}, \alpha_{\mu_{\tau}}\right)\right]^{-3 / 2} .
\end{aligned}
$$


The first term on the RHS of (3.9) is equal to

$$
\begin{array}{r}
s^{-3\left(\mu_{\tau}+1\right)} \ln \left[\sqrt{2} Q\left(P_{\mu_{\tau}}\right)\right]=\gamma_{g}|\tau|^{3 \nu}+s^{-3\left(\mu_{\tau}+1\right)} \ln \frac{\sqrt{\beta \tilde{\Phi}(0)}}{s^{\mu_{\tau}+1}}, \\
\gamma_{g}=c_{\nu}^{3} \bar{\gamma}_{g}, \quad \bar{\gamma}_{g}=\ln \left[\left(\frac{4 s^{7} \bar{u}_{\mu_{\tau}}}{\pi^{4} C\left(h_{\mu_{\tau}}, \alpha_{\mu_{\tau}}\right)}\right)^{1 / 4} I_{0}\left(\eta_{\mu_{\tau}}, \xi_{\mu_{\tau}}\right)\right] .
\end{array}
$$

To find the second term on the RHS of (3.9), we need to calculate $Z_{\mu_{\tau}+1}$. The coefficients occurring in it equal

$$
\begin{aligned}
& d_{\mu_{\tau}+1}(k)=r_{\mu_{\tau}+1} s^{-2\left(\mu_{\tau}+1\right)}+\tilde{q} k^{2}, \quad \tilde{q}=2 \beta \tilde{\Phi}(0) b^{2}, \\
& a_{4}^{\left(\mu_{\tau}+1\right)}=u_{\mu_{\tau}+1} s^{-4\left(\mu_{\tau}+1\right)}, \quad a_{6}^{\left(\mu_{\tau}+1\right)}=w_{\mu_{\tau}+1} s^{-6\left(\mu_{\tau}+1\right)}, \\
& r_{\mu_{\tau}+1}=-\bar{r}_{\mu_{\tau}+1} \beta \tilde{\Phi}(0), \quad \bar{r}_{\mu_{\tau}+1}=2 f_{0}, \\
& u_{\mu_{\tau}+1}=\bar{u}_{\mu_{\tau}+1}(\beta \tilde{\Phi}(0))^{2}, \quad \bar{u}_{\mu_{\tau}+1}=\varphi_{0}-f_{0} \varphi_{0}^{-1 / 2} w_{21}^{(0)}, \\
& w_{\mu_{\tau}+1}=\bar{w}_{\mu_{\tau}+1}(\beta \tilde{\Phi}(0))^{3}, \quad \bar{w}_{\mu_{\tau}+1}=\psi_{0}-f_{0} \varphi_{0} w_{31}^{(0)} .
\end{aligned}
$$

Let us perform the change of variables

$$
\rho_{\boldsymbol{k}}=\rho_{\boldsymbol{k}}^{\prime}+\sqrt{N}\langle\bar{\sigma}\rangle \delta_{\boldsymbol{k}}
$$

in the expression for $Z_{\mu_{\tau}+1}$ in order to extract the free energy related to the ordering that has appeared in the system. Here $\langle\bar{\sigma}\rangle$ is determined from the extremum condition $[17,10]$

$$
\begin{aligned}
\langle\bar{\sigma}\rangle^{2} & =10 \frac{a_{4}^{\left(\mu_{\tau}+1\right)}}{a_{6}^{\left(\mu_{\tau}+1\right)}} \frac{N_{\mu_{\tau}+1}}{N}\left(-1+b_{2}\right) \\
b_{2} & =\sqrt{1+\frac{6 a_{6}^{\left(\mu_{\tau}+1\right)}\left|d_{\mu_{\tau}+1}(0)\right|}{5\left(a_{4}^{\left(\mu_{\tau}+1\right)}\right)^{2}}} .
\end{aligned}
$$

Simultaneously, we include in the treatment a constant external field $h=$ $\mu_{B} \mathcal{H}$, which sustains the separated average moment, and also separate from the sums over $k$ the terms with $k=0$. We obtain

$$
\begin{aligned}
& Z_{\mu_{\tau}+1}=\exp \left(-\beta F_{\sigma}+\beta F_{h}\right) \int \mathrm{d} \rho_{0} \exp \left\{\beta \sqrt{N} h \rho_{0}-\frac{1}{2} \bar{d}_{\mu_{\tau}+1}(0) \rho_{0}^{2}-\right. \\
& \left.-\frac{b_{3}^{\left(\mu_{\tau}+1\right)}}{3 ! \sqrt{N_{\mu_{\tau}+1}}} \rho_{0}^{3}-\frac{b_{4}^{\left(\mu_{\tau}+1\right)}}{4 ! N_{\mu_{\tau}+1}} \rho_{0}^{4}-\frac{b_{5}^{\left(\mu_{\tau}+1\right)}}{5 ! N_{\mu_{\tau}+1} \sqrt{N_{\mu_{\tau}+1}}} \rho_{0}^{5}-\frac{a_{6}^{\left(\mu_{\tau}+1\right)}}{6 ! N_{\mu_{\tau}+1}^{2}} \rho_{0}^{6}\right\} \times \\
& \times \int \exp \left\{-\frac{1}{2} \sum_{k \leqslant B_{\mu_{\tau}+1}}^{\prime} \bar{d}_{\mu_{\tau}+1}(k) \rho_{\boldsymbol{k}} \rho_{-\boldsymbol{k}}\right\} \exp \left\{p_{0}+p_{1} \rho_{0}+p_{2} \rho_{0}^{2}+\right. \\
& \left.+p_{3} \rho_{0}^{3}+p_{4} \rho_{0}^{4}\right\}(\mathrm{d} \rho)^{N_{\mu_{\tau}+1}-1} .
\end{aligned}
$$

The prime on the sum over $k$ means that $k \neq 0$,

$$
-\beta F_{\sigma}=\frac{10}{3}\left|d_{\mu_{\tau}+1}(0)\right| \frac{a_{4}^{\left(\mu_{\tau}+1\right)}}{a_{6}^{\left(\mu_{\tau}+1\right)}} N_{\mu_{\tau}+1}\left(-1+b_{2}\right)-
$$




$$
\begin{aligned}
& -\frac{25}{18} \frac{\left(a_{4}^{\left(\mu_{\tau}+1\right)}\right)^{3}}{\left(a_{6}^{\left(\mu_{\tau}+1\right)}\right)^{2}} N_{\mu_{\tau}+1}\left(-1+b_{2}\right)^{2} \\
& \beta F_{h}=\beta \sqrt{N} h \frac{\sqrt{10 a_{4}^{\left(\mu_{\tau}+1\right)}} \sqrt{N_{\mu_{\tau}+1}}}{\sqrt{a_{6}^{\left(\mu_{\tau}+1\right)}}}\left(-1+b_{2}\right)^{1 / 2} .
\end{aligned}
$$

We have for the integrand coefficients of (3.16)

$$
\begin{aligned}
& \bar{d}_{\mu_{\tau}+1}(k)=4\left|d_{\mu_{\tau}+1}(0)\right|-\frac{10}{3} \frac{\left(a_{4}^{\left(\mu_{\tau}+1\right)}\right)^{2}}{a_{6}^{\left(\mu_{\tau}+1\right)}}\left(-1+b_{2}\right)+\tilde{q} k^{2}= \\
& =c_{\nu}^{2}|\tau|^{2 \nu} \beta \tilde{\Phi}(0)\left[4 \bar{r}_{\mu_{\tau}+1}-\frac{10}{3} \frac{\bar{u}_{\mu_{\tau}+1}^{2}}{\bar{w}_{\mu_{\tau}+1}}\left(-1+b_{2}\right)\right]+\tilde{q} k^{2}, \\
& b_{3}^{\left(\mu_{\tau}+1\right)}=\frac{\sqrt{10} a_{4}^{\left(\mu_{\tau}+1\right)} \sqrt{a_{4}^{\left(\mu_{\tau}+1\right)}}}{\sqrt{a_{6}^{\left(\mu_{\tau}+1\right)}}}\left(-1+b_{2}\right)^{1 / 2}\left[1+\frac{5}{3}\left(-1+b_{2}\right)\right], \\
& b_{4}^{\left(\mu_{\tau}+1\right)}=a_{4}^{\left(\mu_{\tau}+1\right)}\left[1+5\left(-1+b_{2}\right)\right] \\
& b_{5}^{\left(\mu_{\tau}+1\right)}=\sqrt{10} \sqrt{a_{4}^{\left(\mu_{\tau}+1\right)} a_{6}^{\left(\mu_{\tau}+1\right)}}\left(-1+b_{2}\right)^{1 / 2} .
\end{aligned}
$$

The quantities $p_{l}$ are determined by the equations

$$
\begin{aligned}
& p_{0}=-\frac{b_{3}^{\left(\mu_{\tau}+1\right)}}{3 ! \sqrt{N_{\mu_{\tau}+1}}} \sum_{k_{i} \leqslant B_{\mu_{\tau}+1}}^{\prime} \rho_{\boldsymbol{k}_{1}} \rho_{\boldsymbol{k}_{2}} \rho_{\boldsymbol{k}_{3}} \delta_{\boldsymbol{k}_{1}+\boldsymbol{k}_{2}+\boldsymbol{k}_{3}}-\frac{b_{4}^{\left(\mu_{\tau}+1\right)}}{4 ! N_{\mu_{\tau}+1}} \times \\
& \times \sum_{k_{i} \leqslant B_{\mu_{\tau}+1}}^{\prime} \rho_{\boldsymbol{k}_{1}} \cdots \rho_{\boldsymbol{k}_{4}} \delta_{\boldsymbol{k}_{1}+\cdots+\boldsymbol{k}_{4}}-\frac{b_{5}^{\left(\mu_{\tau}+1\right)}}{5 ! N_{\mu_{\tau}+1} \sqrt{N_{\mu_{\tau}+1}}} \times \\
& \times \sum_{k_{i} \leqslant B_{\mu_{\tau}+1}}^{\prime} \rho_{\boldsymbol{k}_{1}} \cdots \rho_{\boldsymbol{k}_{5}} \delta_{\boldsymbol{k}_{1}+\cdots+\boldsymbol{k}_{5}}-\frac{a_{6}^{\left(\mu_{\tau}+1\right)}}{6 ! N_{\mu_{\tau}+1}^{2}} \sum_{k_{i} \leqslant B_{\mu_{\tau}+1}}^{\prime} \rho_{\boldsymbol{k}_{1}} \cdots \rho_{\boldsymbol{k}_{6}} \delta_{\boldsymbol{k}_{1}+\cdots+\boldsymbol{k}_{6}}, \\
& p_{1}=-\frac{b_{3}^{\left(\mu_{\tau}+1\right)}}{2 \sqrt{N_{\mu_{\tau}+1}}} \sum_{k \leqslant B_{\mu_{\tau}+1}}^{\prime} \rho_{\boldsymbol{k}} \rho_{-\boldsymbol{k}}-\frac{b_{4}^{\left(\mu_{\tau}+1\right)}}{3 ! N_{\mu_{\tau}+1}} \sum_{k_{i} \leqslant B_{\mu_{\tau}+1}}^{\prime} \rho_{\boldsymbol{k}_{1}} \rho_{\boldsymbol{k}_{2}} \rho_{\boldsymbol{k}_{3}} \times \\
& \times \delta_{\boldsymbol{k}_{1}+\boldsymbol{k}_{2}+\boldsymbol{k}_{3}}-\frac{b_{5}^{\left(\mu_{\tau}+1\right)}}{4 ! N_{\mu_{\tau}+1} \sqrt{N_{\mu_{\tau}+1}}} \sum_{k_{i} \leqslant B_{\mu_{\tau}+1}}^{\prime} \rho_{\boldsymbol{k}_{1}} \cdots \rho_{\boldsymbol{k}_{4}} \delta_{\boldsymbol{k}_{1}+\cdots+\boldsymbol{k}_{4}}- \\
& -\frac{a_{6}^{\left(\mu_{\tau}+1\right)}}{5 ! N_{\mu_{\tau}+1}^{2}} \sum_{k_{i} \leqslant B_{\mu_{\tau}+1}}^{\prime} \rho_{\boldsymbol{k}_{1}} \cdots \rho_{\boldsymbol{k}_{5}} \delta_{\boldsymbol{k}_{1}+\cdots+\boldsymbol{k}_{5}} \\
& p_{2}=-\frac{b_{4}^{\left(\mu_{\tau}+1\right)}}{4 N_{\mu_{\tau}+1}} \sum_{k \leq B_{\mu_{\tau}+1}}^{\prime} \rho_{\boldsymbol{k}} \rho_{-\boldsymbol{k}}-\frac{b_{5}^{\left(\mu_{\tau}+1\right)}}{12 N_{\mu_{\tau}+1} \sqrt{N_{\mu_{\tau}+1}}} \times \\
& \times \sum_{k_{i} \leqslant B_{\mu_{\tau}+1}}^{\prime} \rho_{\boldsymbol{k}_{1}} \rho_{\boldsymbol{k}_{2}} \rho_{\boldsymbol{k}_{3}} \delta_{\boldsymbol{k}_{1}+\boldsymbol{k}_{2}+\boldsymbol{k}_{3}}-\frac{a_{6}^{\left(\mu_{\tau}+1\right)}}{48 N_{\mu_{\tau}+1}^{2}} \sum_{k_{i} \leqslant B_{\mu_{\tau}+1}}^{\prime} \rho_{\boldsymbol{k}_{1}} \cdots \rho_{\boldsymbol{k}_{4}} \delta_{\boldsymbol{k}_{1}+\cdots+\boldsymbol{k}_{4}}, \\
& p_{3}=-\frac{b_{5}^{\left(\mu_{\tau}+1\right)}}{12 N_{\mu_{\tau}+1} \sqrt{N_{\mu_{\tau}+1}}} \sum_{k \leqslant B_{\mu_{\tau}+1}}^{\prime} \rho_{\boldsymbol{k}} \rho_{-\boldsymbol{k}}-\frac{a_{6}^{\left(\mu_{\tau}+1\right)}}{36 N_{\mu_{\tau}+1}^{2}} \sum_{k_{i} \leqslant B_{\mu_{\tau}+1}}^{\prime} \rho_{\boldsymbol{k}_{1}} \rho_{\boldsymbol{k}_{2}} \rho_{\boldsymbol{k}_{3}} \times
\end{aligned}
$$




$$
\begin{aligned}
& \times \delta_{\boldsymbol{k}_{1}+\boldsymbol{k}_{2}+\boldsymbol{k}_{3}}, \\
& p_{4}=-\frac{a_{6}^{\left(\mu_{\tau}+1\right)}}{48 N_{\mu_{\tau}+1}^{2}} \sum_{k \leqslant B_{\mu_{\tau}+1}}^{\prime} \rho_{\boldsymbol{k}} \rho_{-\boldsymbol{k}} .
\end{aligned}
$$

The dependence of the quantity $4 \bar{r}_{\mu_{\tau}+1}-\frac{10}{3} \frac{\bar{u}_{\mu_{\tau}+1}^{2}}{\bar{w}_{\mu_{\tau}+1}}\left(-1+b_{2}\right)$ on the parameter $s$ is plotted in figure 3 . The dashed line shows the dependence of the analogous quantity $4 f_{0}$ on $s$ for the $\rho^{4}$ model. One can see from figure 3 the

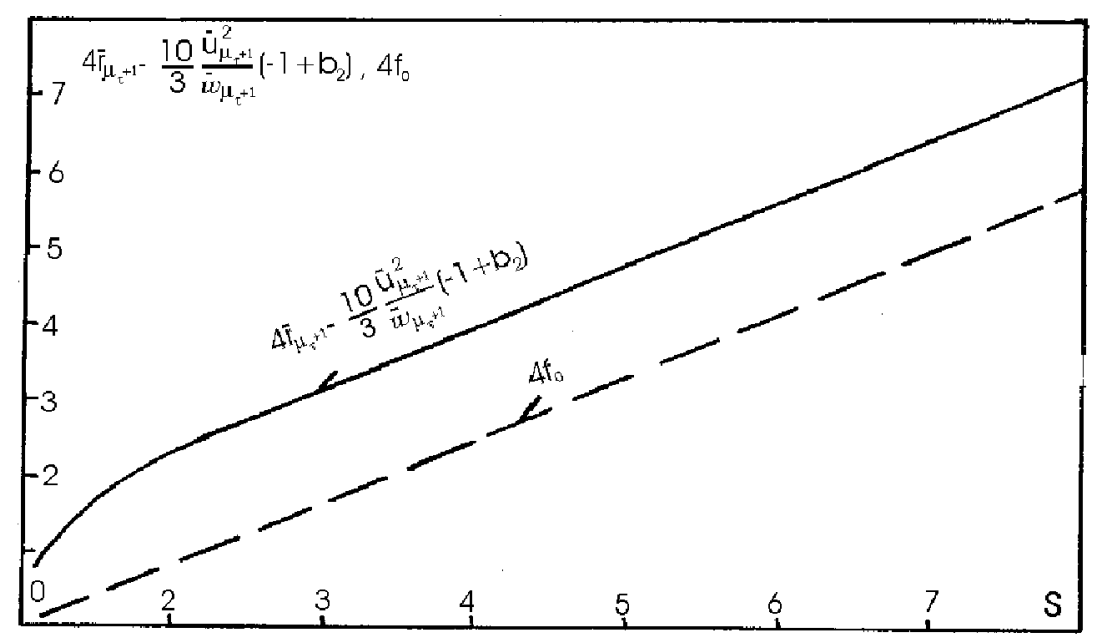

Figure 3. Dependence of the quantities $4 \bar{r}_{\mu_{\tau}+1}-\frac{10}{3} \frac{\bar{u}_{\mu_{\tau}+1}^{2}}{\bar{w}_{\mu_{\tau}+1}}\left(-1+b_{2}\right)$ ( $\rho^{6}$ model) and $4 f_{0}\left(\rho^{4}\right.$ model $)$ on $s$.

quantity $4 \bar{r}_{\mu_{\tau}+1}-\frac{10}{3} \frac{\bar{u}_{\mu_{\tau}+1}^{2}}{\bar{w}_{\mu_{\tau}+1}}\left(-1+b_{2}\right)$ ocurring in $\bar{d}_{\mu_{\tau}+1}(k)(3.18)$ (therefore, and $\left.\bar{d}_{\mu_{\tau}+1}(k)\right)$ is positive for all $s$.

Expanding $\exp \left\{p_{0}+p_{1} \rho_{0}+p_{2} \rho_{0}^{2}+p_{3} \rho_{0}^{3}+p_{4} \rho_{0}^{4}\right\}$ in series and restricting ourselves to the terms of second order, we integrate (3.16) over $\rho_{\boldsymbol{k}}$ with $k \neq 0$ using the Gaussian basis distribution. Gathering up the series over the averages with respect to the Gaussian distribution in the exponential, we obtain [15]

$$
\begin{aligned}
& Z_{\mu_{\tau}+1}=\exp \left(-\beta F_{\sigma}+\beta F_{h}-\beta F_{m}\right) \prod_{k_{i} \leqslant B_{\mu_{\tau}+1}}^{\prime}\left(\frac{\pi}{\bar{d}_{\mu_{\tau}+1}(k)}\right)^{1 / 2} \int \exp \left(\tilde{A}_{1} \rho_{0}+\right. \\
& \left.+\tilde{A}_{2} \rho_{0}^{2}+\tilde{A}_{3} \rho_{0}^{3}+\tilde{A}_{4} \rho_{0}^{4}+\tilde{A}_{5} \rho_{0}^{5}+\tilde{A}_{6} \rho_{0}^{6}+\tilde{A}_{7} \rho_{0}^{7}+\tilde{A}_{8} \rho_{0}^{8}\right) \mathrm{d} \rho_{0},
\end{aligned}
$$

where

$$
\begin{aligned}
-\beta F_{m}= & \frac{1}{4} N_{\mu_{\tau}+1}\left[-\frac{1}{2}\left(b_{4}^{\left(\mu_{\tau}+1\right)}+\frac{a_{6}^{\left(\mu_{\tau}+1\right)}}{6} \mathcal{I}_{1}\right) \mathcal{I}_{1}^{2}+\frac{b_{3}^{\left(\mu_{\tau}+1\right)}}{3} \mathcal{I}_{3} \times\right. \\
& \times\left(b_{3}^{\left(\mu_{\tau}+1\right)}+b_{5}^{\left(\mu_{\tau}+1\right)} \mathcal{I}_{1}\right)+\frac{\left(b_{4}^{\left(\mu_{\tau}+1\right)}\right)^{2}}{4}\left(\frac{\mathcal{I}_{4}}{3}+\mathcal{I}_{1}^{2} \mathcal{I}_{2}\right)+\frac{\left(b_{5}^{\left(\mu_{\tau}+1\right)}\right)^{2}}{12} \times
\end{aligned}
$$




$$
\begin{aligned}
& \times\left(\frac{\mathcal{I}_{5}}{5}+\mathcal{I}_{1}^{2} \mathcal{I}_{3}\right)+\frac{\left(a_{6}^{\left(\mu_{\tau}+1\right)}\right)^{2}}{8}\left(\frac{\mathcal{I}_{6}}{45}+\frac{\mathcal{I}_{1}^{2}}{2}\left(\frac{\mathcal{I}_{1}^{2} \mathcal{I}_{2}}{4}+\frac{\mathcal{I}_{4}}{3}\right)\right)+ \\
& \left.+\frac{b_{4}^{\left(\mu_{\tau}+1\right)} a_{6}^{\left(\mu_{\tau}+1\right)}}{4} \mathcal{I}_{1}\left(\frac{\mathcal{I}_{4}}{3}+\frac{\mathcal{I}_{1}^{2} \mathcal{I}_{2}}{2}\right)\right], \\
& \tilde{A}_{1}=\beta \sqrt{N} h+\frac{1}{2} N_{\mu_{\tau}+1}^{1 / 2}\left[-\mathcal{I}_{1}\left(b_{3}^{\left(\mu_{\tau}+1\right)}+\frac{1}{4} b_{5}^{\left(\mu_{\tau}+1\right)} \mathcal{I}_{1}\right)+\right. \\
& +b_{3}^{\left(\mu_{\tau}+1\right)} b_{4}^{\left(\mu_{\tau}+1\right)}\left(\frac{\mathcal{I}_{3}}{3}+\frac{\mathcal{I}_{1} \mathcal{I}_{2}}{2}\right)+\frac{b_{3}^{\left(\mu_{\tau}+1\right)} a_{6}^{\left(\mu_{\tau}+1\right)}}{2} \mathcal{I}_{1}\left(\frac{\mathcal{I}_{3}}{3}+\frac{\mathcal{I}_{1} \mathcal{I}_{2}}{4}\right)+ \\
& +\frac{b_{4}^{\left(\mu_{\tau}+1\right)} b_{5}^{\left(\mu_{\tau}+1\right)}}{2}\left(\frac{\mathcal{I}_{4}}{6}+\mathcal{I}_{1}\left(\frac{\mathcal{I}_{3}}{3}+\frac{\mathcal{I}_{1} \mathcal{I}_{2}}{2}\right)\right)+\frac{b_{5}^{\left(\mu_{\tau}+1\right)} a_{6}^{\left(\mu_{\tau}+1\right)}}{4}\left(\frac{\mathcal{I}_{5}}{15}+\right. \\
& \left.\left.+\mathcal{I}_{1}\left(\frac{\mathcal{I}_{1} \mathcal{I}_{3}}{3}+\frac{\mathcal{I}_{4}}{6}+\frac{\mathcal{I}_{1}^{2} \mathcal{I}_{2}}{4}\right)\right)\right] \\
& \tilde{A}_{2}=-2\left|d_{\mu_{\tau}+1}(0)\right|+\frac{5}{3} \frac{\left(a_{4}^{\left(\mu_{\tau}+1\right)}\right)^{2}}{a_{6}^{\left(\mu_{\tau}+1\right)}}\left(-1+b_{2}\right)+\frac{1}{4}\left[-\mathcal{I}_{1}\left(b_{4}^{\left(\mu_{\tau}+1\right)}+\right.\right. \\
& \left.+\frac{a_{6}^{\left(\mu_{\tau}+1\right)}}{4} \mathcal{I}_{1}\right)+\left(b_{3}^{\left(\mu_{\tau}+1\right)}\right)^{2} \mathcal{I}_{2}+\left(b_{4}^{\left(\mu_{\tau}+1\right)}\right)^{2}\left(\frac{\mathcal{I}_{3}}{3}+\frac{\mathcal{I}_{1} \mathcal{I}_{2}}{2}\right)+ \\
& +\frac{\left(b_{5}^{\left(\mu_{\tau}+1\right)}\right)^{2}}{2}\left(\frac{\mathcal{I}_{4}}{6}+\mathcal{I}_{1}\left(\frac{\mathcal{I}_{3}}{3}+\frac{\mathcal{I}_{1} \mathcal{I}_{2}}{2}\right)\right)+\frac{\left(a_{6}^{\left(\mu_{\tau}+1\right)}\right)^{2}}{4}\left(\frac{\mathcal{I}_{5}}{15}+\right. \\
& \left.+\mathcal{I}_{1}\left(\frac{\mathcal{I}_{1} \mathcal{I}_{3}}{3}+\frac{\mathcal{I}_{4}}{6}+\frac{\mathcal{I}_{1}^{2} \mathcal{I}_{2}}{4}\right)\right)+b_{3}^{\left(\mu_{\tau}+1\right)} b_{5}^{\left(\mu_{\tau}+1\right)}\left(\frac{\mathcal{I}_{3}}{3}+\mathcal{I}_{1} \mathcal{I}_{2}\right)+ \\
& \left.+b_{4}^{\left(\mu_{\tau}+1\right)} a_{6}^{\left(\mu_{\tau}+1\right)}\left(\frac{\mathcal{I}_{4}}{12}+\mathcal{I}_{1}\left(\frac{\mathcal{I}_{3}}{3}+\frac{3}{8} \mathcal{I}_{1} \mathcal{I}_{2}\right)\right)\right], \\
& \tilde{A}_{3}=\frac{1}{2 N_{\mu_{\tau}+1}^{1 / 2}}\left[-\frac{1}{3}\left(b_{3}^{\left(\mu_{\tau}+1\right)}+\frac{b_{5}^{\left(\mu_{\tau}+1\right)}}{2} \mathcal{I}_{1}\right)+\frac{b_{3}^{\left(\mu_{\tau}+1\right)} a_{6}^{\left(\mu_{\tau}+1\right)}}{2}\left(\frac{\mathcal{I}_{3}}{9}+\right.\right. \\
& \left.+\frac{\mathcal{I}_{1} \mathcal{I}_{2}}{2}\right)+\frac{b_{4}^{\left(\mu_{\tau}+1\right)} b_{5}^{\left(\mu_{\tau}+1\right)}}{2}\left(\frac{\mathcal{I}_{3}}{3}+\frac{2}{3} \mathcal{I}_{1} \mathcal{I}_{2}\right)+\frac{b_{5}^{\left(\mu_{\tau}+1\right)} a_{6}^{\left(\mu_{\tau}+1\right)}}{12}\left(\frac{\mathcal{I}_{4}}{2}+\right. \\
& \left.\left.+\mathcal{I}_{1}\left(\frac{4 \mathcal{I}_{3}}{3}+\frac{7}{4} \mathcal{I}_{1} \mathcal{I}_{2}\right)\right)+\frac{b_{3}^{\left(\mu_{\tau}+1\right)} b_{4}^{\left(\mu_{\tau}+1\right)}}{2} \mathcal{I}_{2}\right] \\
& \tilde{A}_{4}=\frac{1}{4 N_{\mu_{\tau}+1}}\left[-\frac{1}{6}\left(b_{4}^{\left(\mu_{\tau}+1\right)}+\frac{a_{6}^{\left(\mu_{\tau}+1\right)}}{2} \mathcal{I}_{1}\right)+\frac{b_{4}^{\left(\mu_{\tau}+1\right)}}{4} \mathcal{I}_{2}\left(b_{4}^{\left(\mu_{\tau}+1\right)}+\right.\right. \\
& \left.+\frac{7}{6} a_{6}^{\left(\mu_{\tau}+1\right)} \mathcal{I}_{1}\right)+\frac{\left(b_{5}^{\left(\mu_{\tau}+1\right)}\right)^{2}}{6}\left(\frac{\mathcal{I}_{3}}{2}+\mathcal{I}_{1} \mathcal{I}_{2}\right)+\frac{b_{3}^{\left(\mu_{\tau}+1\right)} b_{5}^{\left(\mu_{\tau}+1\right)}}{3} \mathcal{I}_{2}+ \\
& \left.+\frac{a_{6}^{\left(\mu_{\tau}+1\right)} b_{4}^{\left(\mu_{\tau}+1\right)}}{9} \mathcal{I}_{3}+\frac{\left(a_{6}^{\left(\mu_{\tau}+1\right)}\right)^{2}}{6}\left(\frac{\mathcal{I}_{4}}{8}+\mathcal{I}_{1}\left(\frac{\mathcal{I}_{3}}{3}+\frac{7}{16} \mathcal{I}_{1} \mathcal{I}_{2}\right)\right)\right], \\
& \tilde{A}_{5}=\frac{1}{24 N_{\mu_{\tau}+1}^{3 / 2}}\left[b_{5}^{\left(\mu_{\tau}+1\right)}\left(-\frac{1}{5}+b_{4}^{\left(\mu_{\tau}+1\right)} \mathcal{I}_{2}\right)+\frac{a_{6}^{\left(\mu_{\tau}+1\right)} \mathcal{I}_{2}}{2}\left(b_{3}^{\left(\mu_{\tau}+1\right)}+\right.\right. \\
& \left.\left.+\frac{b_{5}^{\left(\mu_{\tau}+1\right)}}{2} \mathcal{I}_{1}\right)+b_{5}^{\left(\mu_{\tau}+1\right)} a_{6}^{\left(\mu_{\tau}+1\right)}\left(\frac{\mathcal{I}_{3}}{3}+\frac{\mathcal{I}_{1} \mathcal{I}_{2}}{3}\right)\right] \\
& \tilde{A}_{6}=\frac{1}{48 N_{\mu_{\tau}+1}^{2}}\left[\frac{a_{6}^{\left(\mu_{\tau}+1\right)}}{3}\left(-\frac{1}{5}+\frac{a_{6}^{\left(\mu_{\tau}+1\right)}}{3} \mathcal{I}_{3}\right)+\frac{\left(b_{5}^{\left(\mu_{\tau}+1\right)}\right)^{2}}{3} \mathcal{I}_{2}+\right.
\end{aligned}
$$




$$
\begin{aligned}
& \left.+\frac{a_{6}^{\left(\mu_{\tau}+1\right)}}{2} \mathcal{I}_{2}\left(b_{4}^{\left(\mu_{\tau}+1\right)}+\frac{a_{6}^{\left(\mu_{\tau}+1\right)}}{2} \mathcal{I}_{1}\right)\right], \\
\tilde{A}_{7}= & \frac{b_{5}^{\left(\mu_{\tau}+1\right)} a_{6}^{\left(\mu_{\tau}+1\right)}}{288 N_{\mu_{\tau}+1}^{5 / 2}} \mathcal{I}_{2}, \\
\tilde{A}_{8}= & \frac{\left(a_{6}^{\left(\mu_{\tau}+1\right)}\right)^{2}}{2304 N_{\mu_{\tau}+1}^{3}} \mathcal{I}_{2} .
\end{aligned}
$$

Expressions for $\mathcal{I}_{m}$ occurring in (3.21) can be found via the transition to the spherical Brillouin zone and integration over $k \in\left(0, B_{\mu_{\tau}+1}\right][17,18]$.

We have

$$
\begin{aligned}
\mathcal{I}_{1}= & \frac{1}{N_{\mu_{\tau}+1}} \sum_{k \leqslant B_{\mu_{\tau}+1}}^{\prime} \frac{1}{\bar{d}_{\mu_{\tau}+1}(k)}=\mathcal{L}\left[4\left|d_{\mu_{\tau}+1}(0)\right|-\frac{10}{3} \times\right. \\
& \left.\times \frac{\left(a_{4}^{\left(\mu_{\tau}+1\right)}\right)^{2}}{a_{6}^{\left(\mu_{\tau}+1\right)}}\left(-1+b_{2}\right)\right]^{-1}
\end{aligned}
$$

or

$$
\mathcal{I}_{1}=\frac{s^{2\left(\mu_{\tau}+1\right)}}{\beta \tilde{\Phi}(0)} \alpha_{1} .
$$

Here,

$$
\begin{aligned}
& \mathcal{L}=3 \frac{x_{r}-\arctan x_{r}}{x_{r}^{3}}, \quad x_{r}=\frac{1}{\sqrt{4 \bar{r}_{\mu_{\tau}+1}-\frac{10}{3} \frac{\bar{u}_{\mu_{\tau}+1}^{2}}{\bar{w}_{\mu_{\tau}+1}}\left(-1+b_{2}\right)}}, \\
& b_{2}=\sqrt{1+\frac{6}{5} \frac{\bar{w}_{\mu_{\tau}+1} \bar{r}_{\mu_{\tau}+1}}{\bar{u}_{\mu_{\tau}+1}^{2}}}, \quad \alpha_{1}=\frac{\mathcal{L}}{4 \bar{r}_{\mu_{\tau}+1}-\frac{10}{3} \frac{\bar{u}_{\mu_{\tau}+1}^{2}}{\bar{w}_{\mu_{\tau}+1}}\left(-1+b_{2}\right)}
\end{aligned}
$$

The quantity $\mathcal{I}_{2}$ can be represented in the form:

$$
\mathcal{I}_{2}=\sum_{r} g^{2}(r)=\frac{s^{4\left(\mu_{\tau}+1\right)}}{(\beta \tilde{\Phi}(0))^{2}} \alpha_{2},
$$

where

$$
\begin{aligned}
g(r)= & \frac{1}{N_{\mu_{\tau}+1}} \sum_{k \leqslant B_{\mu_{\tau}+1}}^{\prime} \frac{\mathrm{e}^{i \boldsymbol{k} \boldsymbol{r}}}{\bar{d}_{\mu_{\tau}+1}(k)}=\frac{6 s^{2\left(\mu_{\tau}+1\right)}}{\beta \tilde{\Phi}(0)\left(B_{\mu_{\tau}+1} r\right)^{3}}\left\{8 \bar{r}_{\mu_{\tau}+1}-\right. \\
& \left.-\frac{20}{3} \frac{\bar{u}_{\mu_{\tau}+1}^{2}}{\bar{w}_{\mu_{\tau}+1}}\left(-1+b_{2}\right)+1\right\}^{-1}\left[\sin \left(B_{\mu_{\tau}+1} r\right)-B_{\mu_{\tau}+1} r \cos \left(B_{\mu_{\tau}+1} r\right)\right], \\
\alpha_{2}= & \alpha_{1}^{2}+6 e_{1}^{2}\left(1+e_{2}^{2}\right), \\
e_{1}= & \frac{6}{\pi^{2}\left[8 \bar{r}_{\mu_{\tau}+1}-\frac{20}{3} \frac{\bar{u}_{\mu_{\tau}+1}^{2}}{\bar{w}_{\mu_{\tau}+1}}\left(-1+b_{2}\right)+1\right]}, \\
e_{2}= & \frac{1}{2 \pi}[\sin (\pi \sqrt{2})-\pi \sqrt{2} \cos (\pi \sqrt{2})] \approx 0.034861 .
\end{aligned}
$$


The other $\mathcal{I}_{l}(l=3,4,5,6)$ are determined by analogous relations

$$
\mathcal{I}_{l}=\sum_{r} g^{l}(r)=\frac{s^{2 l\left(\mu_{\tau}+1\right)}}{(\beta \tilde{\Phi}(0))^{l}} \alpha_{l}
$$

with

$$
\alpha_{l}=\alpha_{1}^{l}+6 e_{1}^{l}\left(1+\frac{e_{2}^{l}}{2^{l / 2-1}}\right)
$$

The values of $\alpha_{m}(m=1,2,3,4,5,6)$ are given in table 6 .

Table 6. Numerical values of $\alpha_{m}$.

\begin{tabular}{llccccc}
\hline \multicolumn{1}{c}{$s$} & $\alpha_{1}$ & $\alpha_{2}$ & $\alpha_{3}$ & $\alpha_{4}$ & $\alpha_{5}$ & $\alpha_{6}$ \\
\hline \multirow{2}{*}{2} & 0.3456 & 0.1893 & 0.0488 & 0.0151 & 0.0050 & 0.0017 \\
2.5 & 0.2981 & 0.1406 & 0.0313 & 0.0083 & 0.0024 & 0.0007 \\
2.7349 & 0.2821 & 0.1258 & 0.0265 & 0.0067 & 0.0018 & 0.0005 \\
3 & 0.2664 & 0.1122 & 0.0223 & 0.0053 & 0.0014 & 0.0004 \\
3.5 & 0.2412 & 0.0918 & 0.0165 & 0.0036 & 0.0008 & 0.0002 \\
3.5862 & 0.2373 & 0.0888 & 0.0158 & 0.0033 & 0.0008 & 0.0002 \\
4 & 0.2201 & 0.0764 & 0.0126 & 0.0025 & 0.0005 & 0.0001 \\
& & & & & & \\
\hline
\end{tabular}

Let us perform the change of the variable $\rho_{0}$ in $(3.20)$

$$
\rho_{0}=\rho_{0}^{\prime}-\sqrt{N}\langle\bar{\sigma}\rangle,
$$

which cancels terms proportional to odd powers of $\rho_{0}$ in the exponent of the integrand. We obtain

$$
\begin{aligned}
Z_{\mu_{\tau}+1} & =\exp \left(-\beta F_{\mu_{\tau}+1}^{\prime}\right) \int \exp \left[\beta \sqrt{N} \rho_{0} h+\tilde{B} \rho_{0}^{2}-\frac{G}{N} \rho_{0}^{4}-\right. \\
& \left.-\frac{D}{N^{2}} \rho_{0}^{6}\right] \mathrm{d} \rho_{0},
\end{aligned}
$$

where

$$
\begin{aligned}
-\beta F_{\mu_{\tau}+1}^{\prime}= & N_{\mu_{\tau}+1}\left\{\frac{5}{2} \bar{r}_{\mu_{\tau}+1}\left(\alpha_{1}+\frac{5}{2} \bar{r}_{\mu_{\tau}+1} \alpha_{2}\right)-\right. \\
& -\frac{\alpha_{1}^{2}}{8}\left(\bar{u}_{\mu_{\tau}+1}+\frac{\bar{w}_{\mu_{\tau}+1}}{6} \alpha_{1}\right)+\frac{\bar{u}_{\mu_{\tau}+1}^{2}}{8}\left[\frac{\alpha_{4}}{6}+\alpha_{1}^{2} \alpha_{2}\left(\frac{1}{2}+\right.\right. \\
& \left.\left.+\frac{5}{3}\left(-1+b_{2}\right)\right)\right]-\frac{5}{4} \bar{r}_{\mu_{\tau}+1} \alpha_{1} \alpha_{2}\left[\bar{u}_{\mu_{\tau}+1}+\frac{\bar{w}_{\mu_{\tau}+1}}{4} \alpha_{1}\right]+ \\
& +\frac{\bar{w}_{\mu_{\tau}+1}^{2}}{32}\left[\frac{\alpha_{6}}{45}+\frac{\alpha_{1}^{2}}{2}\left(\frac{\alpha_{4}}{3}+\frac{\alpha_{1}^{2} \alpha_{2}}{4}\right)\right]+ \\
& +\frac{\bar{u}_{\mu_{\tau}+1} \bar{w}_{\mu_{\tau}+1} \alpha_{1}}{16}\left[\frac{\alpha_{4}}{3}+\frac{\alpha_{1}^{2} \alpha_{2}}{2}\right]-
\end{aligned}
$$




$$
\begin{aligned}
& -\frac{5}{3} \frac{\bar{u}_{\mu_{\tau}+1}^{2}}{\bar{w}_{\mu_{\tau}+1}}\left[\left(-1+b_{2}\right)\left(\alpha_{1}-\bar{u}_{\mu_{\tau}+1} \alpha_{2}\left(\frac{\alpha_{1}}{2}-\frac{10}{3} \frac{\bar{u}_{\mu_{\tau}+1}}{\bar{w}_{\mu_{\tau}+1}}\right)\right)-\right. \\
& \left.-\left(7-5 b_{2}\right) \bar{r}_{\mu_{\tau}+1} \alpha_{2}\right]-\frac{1}{2} \ln \left(\frac{\beta \tilde{\Phi}(0)}{s^{2\left(\mu_{\tau}+1\right)}}\right)-\frac{1}{2} \ln [(1+ \\
& \left.\left.\left.+4 \bar{r}_{\mu_{\tau}+1}-\frac{10}{3} \frac{\bar{u}_{\mu_{\tau}+1}^{2}}{\bar{w}_{\mu_{\tau}+1}}\left(-1+b_{2}\right)\right) \pi^{-1}\right]+\frac{1}{3}-\frac{\mathcal{L}}{3}\right\}, \\
& \tilde{B}=\frac{1}{2} c_{\nu}^{2}|\tau|^{2 \nu} \beta \tilde{\Phi}(0) \bar{r}_{\mu_{\tau}+1} \bar{B}, \\
& G=c_{\nu}|\tau|^{\nu}(\beta \tilde{\Phi}(0))^{2} s_{0}^{3} \bar{G}, \\
& D=(\beta \tilde{\Phi}(0))^{3} s_{0}^{6} \bar{D} \text {, } \\
& \bar{B}=1-\frac{\alpha_{1}}{2 \bar{r}_{\mu_{\tau}+1}}\left(\bar{u}_{\mu_{\tau}+1}+\frac{\bar{w}_{\mu_{\tau}+1}}{4} \alpha_{1}\right)+\frac{\bar{u}_{\mu_{\tau}+1}^{2}}{\bar{r}_{\mu_{\tau}+1}}\left[\frac{5}{3}\left(-1+b_{2}\right) \alpha_{2} \times\right. \\
& \left.\times\left(\frac{\alpha_{1}}{2}+\frac{\bar{u}_{\mu_{\tau}+1}}{\bar{w}_{\mu_{\tau}+1}}\right)+\frac{1}{2}\left(\frac{\alpha_{1} \alpha_{2}}{2}+\frac{\alpha_{3}}{3}\right)\right]-\frac{5}{2} \alpha_{2}\left(\bar{u}_{\mu_{\tau}+1}+\right. \\
& \left.+\frac{\bar{w}_{\mu_{\tau}+1} \alpha_{1}}{2}\right)+\frac{\bar{w}_{\mu_{\tau}+1}^{2}}{8 \bar{r}_{\mu_{\tau}+1}}\left[\frac{\alpha_{5}}{15}+\alpha_{1}\left(\frac{\alpha_{1} \alpha_{3}}{3}+\frac{1}{2}\left(\frac{\alpha_{4}}{3}+\frac{\alpha_{1}^{2} \alpha_{2}}{2}\right)\right)\right]+ \\
& +\frac{\bar{u}_{\mu_{\tau}+1} \bar{w}_{\mu_{\tau}+1}}{2 \bar{r}_{\mu_{\tau}+1}}\left[\frac{\alpha_{4}}{12}+\alpha_{1}\left(\frac{3 \alpha_{1} \alpha_{2}}{8}+\frac{\alpha_{3}}{3}\right)\right] \\
& \bar{G}=\frac{1}{24}\left[\bar{u}_{\mu_{\tau}+1}+\frac{\bar{w}_{\mu_{\tau}+1}}{2}\left(\alpha_{1}+5 \bar{r}_{\mu_{\tau}+1} \alpha_{2}\right)\right]-\frac{\bar{u}_{\mu_{\tau}+1}^{2} \alpha_{2}}{8}\left[\frac{1}{2}+\frac{5}{9}(-1+\right. \\
& \left.\left.+b_{2}\right)\right]-\frac{\bar{u}_{\mu_{\tau}+1} \bar{w}_{\mu_{\tau}+1}}{12}\left(\frac{7}{8} \alpha_{1} \alpha_{2}+\frac{\alpha_{3}}{3}\right)-\frac{\bar{w}_{\mu_{\tau}+1}^{2}}{24}\left[\frac{\alpha_{4}}{8}+\right. \\
& \left.+\alpha_{1}\left(\frac{7}{16} \alpha_{1} \alpha_{2}+\frac{\alpha_{3}}{3}\right)\right] \text {, } \\
& \bar{D}=\frac{\bar{w}_{\mu_{\tau}+1}}{48}\left(\frac{1}{15}-\frac{\bar{u}_{\mu_{\tau}+1}}{2} \alpha_{2}\right)-\frac{\bar{w}_{\mu_{\tau}+1}^{2}}{48}\left(\frac{\alpha_{3}}{9}+\frac{\alpha_{1} \alpha_{2}}{4}\right) \text {. }
\end{aligned}
$$

The quantities $\bar{B}, \bar{G}, \bar{D}$ are given in table 7 .

Table 7 . Values of $\bar{B}, \bar{G}, \bar{D},\langle\bar{\sigma}\rangle^{(0)}, \Gamma^{+}, \Gamma^{-}$for different $s$.

\begin{tabular}{lllllll}
\hline \multicolumn{1}{c}{$s$} & $\bar{B}$ & $\bar{G}$ & $\bar{D}$ & $\langle\bar{\sigma}\rangle^{(0)}$ & $\Gamma^{+}$ & $\Gamma^{-}$ \\
& & & & & & \\
\hline 2 & 0.8296 & 0.0153 & 0.0002 & 3.0942 & 2.6052 & 0.2970 \\
2.5 & 0.8289 & 0.0206 & 0.0003 & 3.0031 & 3.8147 & 0.3121 \\
2.7349 & 0.8277 & 0.0231 & 0.0003 & 2.9669 & 2.9709 & 0.3211 \\
3 & 0.8267 & 0.0260 & 0.0003 & 2.9309 & 3.3248 & 0.3318 \\
3.5 & 0.8266 & 0.0316 & 0.0003 & 2.8730 & 4.0679 & 0.3528 \\
3.5862 & 0.8268 & 0.0326 & 0.0003 & 2.8640 & 4.2071 & 0.3565 \\
4 & 0.8282 & 0.0376 & 0.0004 & 2.8238 & 4.9254 & 0.3748 \\
& & & & & & \\
\hline
\end{tabular}


Using the method of the steepest descent for $Z_{\mu_{\tau}+1}$ (3.29), we find

$$
Z_{\mu_{\tau}+1}=\sqrt{\frac{2 \pi}{E_{0}^{\prime \prime}(\bar{\rho})}} \exp \left[-\beta F_{\mu_{\tau}+1}^{\prime}-N E_{0}(\bar{\rho})\right] .
$$

Here $\bar{\rho}$ is the extremum point of the expression

$$
E_{0}(\rho)=D \rho^{6}+G \rho^{4}-\tilde{B} \rho^{2}-\beta h \rho
$$

arising in the exponential of the integrand of (3.29) at the substitution

$$
\rho_{0}=\sqrt{N} \rho .
$$

For $E_{0}(\bar{\rho})$ at $h=0$ we obtain

$$
\begin{gathered}
E_{0}(\bar{\rho})=-s^{-3\left(\mu_{\tau}+1\right)} s_{0}^{-3} \bar{E}_{0} \\
\bar{E}_{0}=\frac{2 \bar{G}^{3}}{27 \bar{D}^{2}}\left(-1+\sqrt{1+\frac{3}{2} \frac{\bar{r}_{\mu_{\tau}+1} \bar{B} \bar{D}}{\bar{G}^{2}}}\right)+\frac{\bar{r}_{\mu_{\tau}+1} \bar{B} \bar{G}}{6 \bar{D}} \times \\
\times\left(-1+\frac{2}{3} \sqrt{1+\frac{3}{2} \frac{\bar{r}_{\mu_{\tau}+1} \bar{B} \bar{D}}{\bar{G}^{2}}}\right) .
\end{gathered}
$$

Having (3.12) and (3.31), we can calculate the contribution to the system free energy at $T<T_{c}$ from the long-wave phases of the spin moment density fluctuations (3.9):

$$
\begin{aligned}
F_{I G R}= & -k T N^{\prime}\left(\gamma_{3}^{\left(\mu_{\tau}\right)}+\gamma_{3}^{\langle\sigma\rangle}\right)|\tau|^{3 \nu}, \\
\gamma_{3}^{\left(\mu_{\tau}\right)=} & \gamma_{g}+\gamma_{\rho}=c_{\nu}^{3} \bar{\gamma}_{3}^{\left(\mu_{\tau}\right)}, \quad \bar{\gamma}_{3}^{\left(\mu_{\tau}\right)}=\bar{\gamma}_{g}+\bar{\gamma}_{\rho}, \\
\gamma_{\rho}= & c_{\nu}^{3} \bar{\gamma}_{\rho}, \quad \bar{\gamma}_{\rho}=\frac{5}{2} \bar{r}_{\mu_{\tau}+1}\left(\alpha_{1}+\frac{5}{2} \bar{r}_{\mu_{\tau}+1} \alpha_{2}\right)-\frac{\alpha_{1}^{2}}{8} \times \\
& \times\left(\bar{u}_{\mu_{\tau}+1}+\frac{\bar{w}_{\mu_{\tau}+1}}{6} \alpha_{1}\right)+\frac{\bar{u}_{\mu_{\tau}+1}^{2}}{8}\left[\frac{\alpha_{4}}{6}+\alpha_{1}^{2} \alpha_{2}\left(\frac{1}{2}+\frac{5}{3}\left(-1+b_{2}\right)\right)\right]- \\
& -\frac{5}{4} \bar{r}_{\mu_{\tau}+1} \alpha_{1} \alpha_{2}\left[\bar{u}_{\mu_{\tau}+1}+\frac{\bar{w}_{\mu_{\tau}+1}}{4} \alpha_{1}\right]+\frac{\bar{w}_{\mu_{\tau}+1}^{2}}{32}\left[\frac{\alpha_{6}}{45}+\frac{\alpha_{1}^{2}}{2} \times\right. \\
& \left.\times\left(\frac{\alpha_{4}}{3}+\frac{\alpha_{1}^{2} \alpha_{2}}{4}\right)\right]+\frac{\bar{u}_{\mu_{\tau}+1} \bar{w}_{\mu_{\tau}+1} \alpha_{1}}{16}\left[\frac{\alpha_{4}}{3}+\frac{\alpha_{1}^{2} \alpha_{2}}{2}\right]-\frac{5}{3} \frac{\bar{u}_{\mu_{\tau}+1}^{2}}{\bar{w}_{\mu_{\tau}+1}} \times \\
& \times\left[\left(-1+b_{2}\right)\left(\alpha_{1}-\bar{u}_{\mu_{\tau}+1} \alpha_{2}\left(\frac{\alpha_{1}}{2}-\frac{10}{3} \frac{\bar{u}_{\mu_{\tau}+1}}{\bar{w}_{\mu_{\tau}+1}}\right)\right)-\left(7-5 b_{2}\right) \times\right. \\
& \left.\times \bar{r}_{\mu_{\tau}+1} \alpha_{2}\right]-\frac{1}{2} \ln \left[\frac{1+4 \bar{r}_{\mu_{\tau}+1}-\frac{10}{3} \frac{\bar{u}_{\mu_{\tau}+1}^{2}}{\bar{w}_{\mu_{\tau}+1}}\left(-1+b_{2}\right)}{\pi}\right]+\frac{1}{3}-\frac{\mathcal{L}}{3}, \\
\gamma_{3}^{\langle\sigma\rangle}= & c_{\nu}^{3} \bar{\gamma}_{3}^{\langle\sigma\rangle}, \quad \bar{\gamma}_{3}^{\langle\sigma\rangle}=\bar{E}_{0} .
\end{aligned}
$$

The quantity $\gamma_{3}^{\mu_{\tau}}$ determines the free energy after the exit from the CR region, and $\gamma_{3}^{\langle\sigma\rangle}$ determines the free energy of the ordering. The values of $\bar{\gamma}_{g}, \bar{\gamma}_{\rho}, \bar{\gamma}_{3}^{\left(\mu_{\tau}\right)}, \bar{\gamma}_{3}^{\langle\sigma\rangle}$ are given in table 8 . 
Table 8. Values of $\bar{\gamma}_{g}, \bar{\gamma}_{\rho}, \bar{\gamma}_{3}^{\left(\mu_{\tau}\right)}, \bar{\gamma}_{3}^{\langle\sigma\rangle}$.

\begin{tabular}{lcccc}
\hline \multicolumn{1}{c}{$s$} & $\bar{\gamma}_{g}$ & $\bar{\gamma}_{\rho}$ & $\bar{\gamma}_{3}^{\left(\mu_{\tau}\right)}$ & $\bar{\gamma}_{3}^{<\sigma>}$ \\
\hline 2 & -0.3024 & 1.0386 & 0.7362 & 1.7618 \\
2.5 & -0.0869 & 1.0269 & 0.9399 & 2.0456 \\
2.7349 & 0.0039 & 1.0227 & 1.0265 & 2.1572 \\
3 & 0.0986 & 1.0179 & 1.1164 & 2.2808 \\
3.5 & 0.2579 & 1.0076 & 1.2655 & 2.5154 \\
3.5862 & 0.2832 & 1.0056 & 1.2888 & 2.5563 \\
4 & 0.3967 & 0.9955 & 1.3922 & 2.7538 \\
& & & & \\
\hline
\end{tabular}

The entropy, internal energy and specific heat of the system corresponding to the IGR region read

$$
\begin{aligned}
& S_{I G R}=S_{\mu_{\tau}}+S_{\langle\sigma\rangle}, \quad U_{I G R}=U_{\mu_{\tau}}+U_{\langle\sigma\rangle}, \quad C_{I G R}=C_{\mu_{\tau}}+C_{\langle\sigma\rangle}, \\
& S_{\eta}=-k N^{\prime}|\tau|^{1-\alpha} u_{3}^{(\eta)}, \quad U_{\eta}=-k T N^{\prime}|\tau|^{1-\alpha} u_{3}^{(\eta)}, \\
& C_{\eta}=k N^{\prime} c_{3}^{(\eta)}|\tau|^{-\alpha}, \quad u_{3}^{(\eta)}=3 \nu \gamma_{3}^{(\eta)}, \quad c_{3}^{(\eta)}=3 \nu(3 \nu-1) \gamma_{3}^{(\eta)} .
\end{aligned}
$$

The index $\eta$ can take two values: $\mu_{\tau}$ and $\langle\sigma\rangle$.

\section{The order parameter of the system}

The mean spin moment is the order parameter of the model investigated. It is related to the presence of the nonzero value of the $\mathrm{CV} \rho_{0}$ at which the integrand of (3.29) has an extremum. Performing the substitution (3.33) in that integrand, we obtain

$$
Z_{\mu_{\tau}+1}=e^{-\beta F_{\mu_{\tau}+1}^{\prime}} \sqrt{N} \int \mathrm{e}^{-N E_{0}(\rho)} \mathrm{d} \rho,
$$

where $E_{0}(\rho)$ is given in (3.32). Owing to the factor $N$ in the exponent in (4.1), the integrand has a sharp maximum at the point $\bar{\rho}$ corresponding to the equilibrium value of the order parameter. The value of $\bar{\rho}$ can be found from the extremum condition $\frac{\partial E_{0}(\rho)}{\partial \rho}=0$ or

$$
6 D \bar{\rho}^{5}+4 G \bar{\rho}^{3}-2 \tilde{B} \bar{\rho}-\beta h=0 .
$$

In the case $h=0$ we obtain a biquadratic equation, which is reduced by means of substitution

$$
\bar{\rho}^{2}=y
$$

to the equation

$$
6 D y^{2}+4 G y-2 \tilde{B}=0 .
$$


Extracting the temperature dependence, we obtain an equation for the mean spin moment $\langle\sigma\rangle=\bar{\rho}=\sqrt{y}$ :

$$
\begin{aligned}
\langle\sigma\rangle & =|\tau|^{\beta}\langle\sigma\rangle^{(0)}, \quad \beta=\nu / 2, \\
\langle\sigma\rangle^{(0)} & =c_{\nu}^{1 / 2}(\beta \tilde{\Phi}(0))^{-1 / 2} s_{0}^{-3 / 2}\langle\bar{\sigma}\rangle^{(0)}, \\
\langle\bar{\sigma}\rangle^{(0)} & =\left[\frac{\bar{G}}{3 \bar{D}}\left(-1+\sqrt{1+\frac{3}{2} \frac{\bar{r}_{\mu_{\tau}+1} \bar{B} \bar{D}}{\bar{G}^{2}}}\right)\right]^{1 / 2} .
\end{aligned}
$$

The value of $\langle\bar{\sigma}\rangle^{(0)}$ is given in table 7 .

The susceptibility per particle $\chi$ can be found from equation $(4.2)$ by differentiating it with respect to $\mathcal{H}$ and using the relation $\chi=\mu_{B} \frac{\partial\langle\sigma\rangle}{\partial \mathcal{H}}$ :

$$
\chi=\frac{\beta \mu_{B}^{2}}{30 D \bar{\rho}^{4}+12 G \bar{\rho}^{2}-2 \tilde{B}} .
$$

Separating the temperature dependence in the coefficients $D, G, \tilde{B}$ (see $(3.30)$ ), we obtain a final expression for the susceptibility.

\section{Thermodynamics of the system in the vicinity of the phase tran- sition point}

Having calculated the contributions to the system free energy from the short-wave and long-wave modes of the spin moment density oscillations both above and below $T_{c}$, we can compute the total free energy

$$
F= \begin{cases}F_{0}+F_{C R}+F_{L G R}, & T>T_{c}, \\ F_{0}+F_{C R}+F_{I G R}, & T<T_{c},\end{cases}
$$

the entropy, internal energy and specific heat.

We obtain the total free energy of the system at $h=0$ taking (2.17), (2.23) and (3.6), (3.35) into account:

$$
F=\left\{\begin{array}{l}
-k T N^{\prime}\left[\gamma_{0}+\gamma_{1} \tau+\gamma_{2} \tau^{2}+\gamma_{3}^{+} \tau^{3 \nu}\right], \quad T>T_{c}, \quad T<T_{c}, \\
-k T N^{\prime}\left[\gamma_{0}+\gamma_{1}|\tau|+\gamma_{2}|\tau|^{2}+\gamma_{3}^{-}|\tau|^{3 \nu}\right], \quad T
\end{array}\right.
$$

where (see table 9)

$$
\begin{aligned}
& \gamma_{0}=\gamma_{0}^{(C R)}+s_{0}^{3} \ln 2, \\
& \gamma_{3}^{+}=-\gamma_{3}^{(C R)+}+f_{L G R}, \\
& \gamma_{3}^{-}=-\gamma_{3}^{(C R)-}+\gamma_{I G R}, \quad \gamma_{I G R}=\gamma_{3}^{\left(\mu_{\tau}\right)}+\gamma_{3}^{\langle\sigma\rangle} .
\end{aligned}
$$

The coefficients $\gamma_{3}^{ \pm}$can be written as a product of the universal part $\bar{\gamma}_{3}^{ \pm}$(table 9) and non-universal factor $c_{\nu}^{3}$ depending on the microscopic parameters of the Hamiltonian (the lattice constant $c$, the effective interaction radius $b$ and the value $\tilde{\Phi}(0)$ of the interaction potential Fourier transform at $k=0$ ):

$$
\begin{aligned}
& \gamma_{3}^{ \pm}=c_{\nu}^{3} \bar{\gamma}_{3}^{ \pm}, \\
& \bar{\gamma}_{3}^{+}=-\gamma^{+}+\bar{f}_{L G R}=-\gamma_{+}+\bar{f}_{\text {ПО }}+\bar{f}^{\prime}, \\
& \bar{\gamma}_{3}^{-}=-\gamma^{-}+\bar{\gamma}_{g}+\bar{\gamma}_{\rho}+\bar{\gamma}_{3}^{\langle\sigma\rangle} .
\end{aligned}
$$


Table 9. Coefficients $\gamma_{0}, \gamma_{3}^{ \pm}$and $\bar{\gamma}_{3}^{ \pm}$.

\begin{tabular}{llllll}
\hline \multicolumn{1}{c}{$s$} & $\gamma_{0}$ & $\bar{\gamma}_{3}^{+}$ & $\gamma_{3}^{+}$ & $\bar{\gamma}_{3}^{-}$ & $\gamma_{3}^{-}$ \\
\hline 2 & & & & & \\
2.5 & 61.1798 & 0.9699 & 2.9033 & 1.7599 & 5.2680 \\
2.7349 & 61.1930 & 1.5898 & 3.2734 & 2.4612 & 5.0675 \\
3 & 61.1999 & 2.8654 & 3.3020 & 2.7650 & 4.8944 \\
3.5 & 61.2150 & 2.8206 & 3.2783 & 3.1073 & 4.6793 \\
3.5862 & 61.2179 & 2.9445 & 3.1704 & 3.8086 & 4.3013 \\
4 & 61.2325 & 3.6167 & 3.1179 & 4.6608 & 4.2448 \\
& & & & & \\
\hline
\end{tabular}

We have for the entropy, internal energy and specific heat of the system

$$
\begin{gathered}
S=\left\{\begin{array}{l}
k N^{\prime}\left[s^{(0)}+c_{0} \tau+u_{3}^{+} \tau^{1-\alpha}\right], \quad T>T_{c}, \\
k N^{\prime}\left[s^{(0)}-c_{0}|\tau|-u_{3}^{-}|\tau|^{1-\alpha}\right], \quad T<T_{c},
\end{array}\right. \\
U=\left\{\begin{array}{l}
k T N^{\prime}\left[\gamma_{1}+u_{1} \tau+u_{3}^{+} \tau^{1-\alpha}\right], T>T_{c}, \\
k T N^{\prime}\left[\gamma_{1}-u_{1}|\tau|-u_{3}^{-}|\tau|^{1-\alpha}\right], \quad T<T_{c},
\end{array}\right. \\
C=\left\{\begin{array}{l}
k N^{\prime}\left[c_{0}+c_{3}^{+} \tau^{-\alpha}\right], T>T_{c}, \\
k N^{\prime}\left[c_{0}+c_{3}^{-}|\tau|^{-\alpha}\right], T<T_{c}
\end{array}\right.
\end{gathered}
$$

with coefficients given by the relations

$$
\begin{aligned}
& s^{(0)}=\gamma_{0}+\gamma_{1}, \\
& u_{3}^{ \pm}=c_{\nu}^{3} \bar{u}_{3}^{ \pm}, \quad \bar{u}_{3}^{ \pm}=3 \nu \bar{\gamma}_{3}^{ \pm}, \\
& u_{1}=2 \gamma_{2}+\gamma_{1}, \\
& c_{3}^{ \pm}=c_{\nu}^{3} \bar{c}_{3}^{ \pm}, \quad \bar{c}_{3}^{ \pm}=3 \nu(3 \nu-1) \bar{\gamma}_{3}^{ \pm} .
\end{aligned}
$$

The formula for the specific heat can also be rewritten as

$$
\begin{gathered}
C / k N^{\prime}=\frac{A^{ \pm}}{\alpha}|\tau|^{-\alpha}+B^{ \pm}, \\
A^{ \pm}=c_{\nu}^{3} \alpha \bar{c}_{3}^{ \pm}, \quad B^{ \pm}=c_{0} .
\end{gathered}
$$

The plus and minus signs correspond to $T>T_{c}$ and $T<T_{c}$, respectively.

The system susceptibility per particle at infinitely small values of the external field $\mathcal{H}$ at $T>T_{c}\left(\chi=-\frac{1}{N} \frac{\partial^{2} F_{L G R}}{\partial \mathcal{H}^{2}}\right.$, see (2.23)) and $T<T_{c}$ (see $(4.6))$ is given by

$$
\chi=\left\{\begin{array}{l}
\Gamma^{+} \tau^{-\gamma} \frac{\mu_{B}^{2}}{\Phi(0)}, \quad T>T_{c}, \\
\Gamma^{-}|\tau|^{-\gamma} \frac{\mu_{B}^{2}}{\Phi(0)}, \quad T<T_{c} .
\end{array}\right.
$$




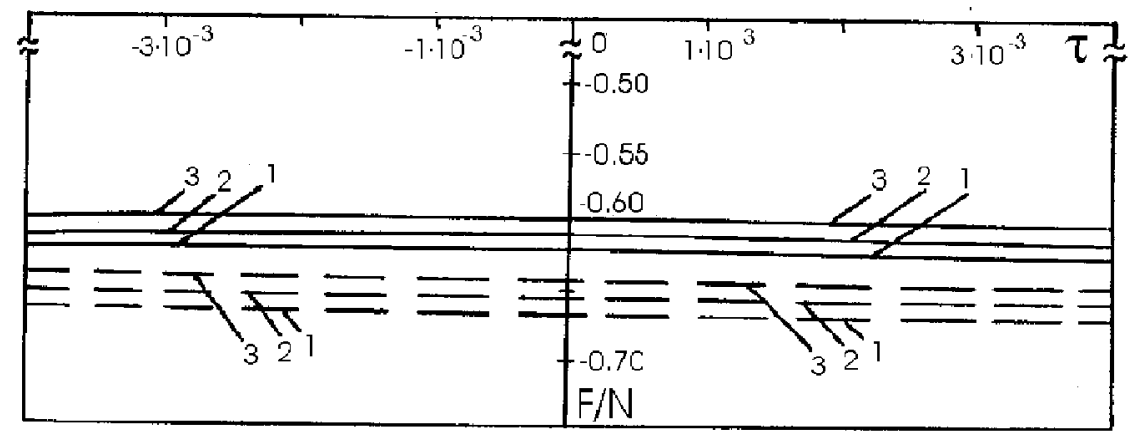

Figure 4. Temperature dependence of the free energy of the system for different values of the RG parameter $s$ within the frames of the $\rho^{6}$ model (solid lines). For comparison we show the free energy of the system in the quartic basis distribution approximation with allowance for confluent corrections [9] (dashed lines). (1) $s=2,(2) s=2.5,(3) s=3$.

Here, (see table 7),

$$
\begin{aligned}
\Gamma^{+} & =2 c_{\nu}^{-2} \bar{\gamma}_{4}^{+}, \\
\Gamma^{-} & =c_{\nu}^{-2}\left\{\frac{10}{3} \frac{\bar{G}^{2}}{\bar{D}}\left(-1+\sqrt{1+\frac{3}{2} \frac{\bar{r}_{\mu_{\tau}+1} \bar{B} \bar{D}}{\bar{G}^{2}}}\right)\left(\frac{1}{5}+\sqrt{1+\frac{3}{2} \frac{\bar{r}_{\mu_{\tau}+1} \bar{B} \bar{D}}{\bar{G}^{2}}}\right)-\right. \\
& \left.-\bar{r}_{\mu_{\tau}+1} \bar{B}\right\}^{-1} \\
\gamma & =2 \nu .
\end{aligned}
$$

Plots of the temperature dependence of the system free energy $F / N$, entropy $S / k N$, specific heat $C / k N$, mean spin moment $\langle\sigma\rangle(4.5)$, susceptibility $\chi(5.7)$ at $s=2,2.5,3$ are shown in figures $4-8$.

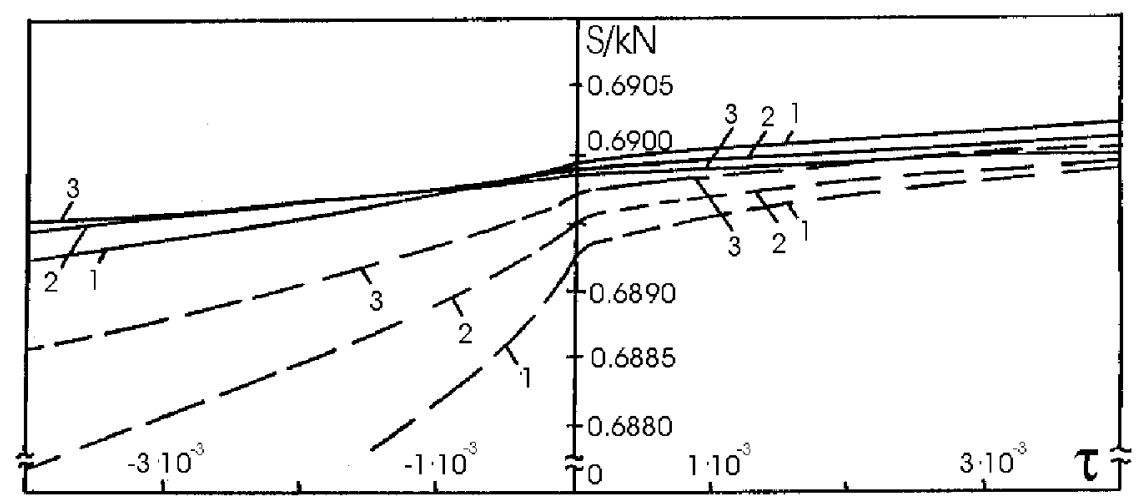

Figure 5. Dependence of the system entropy on $\tau$ (Notations are the same as in figure 4). 


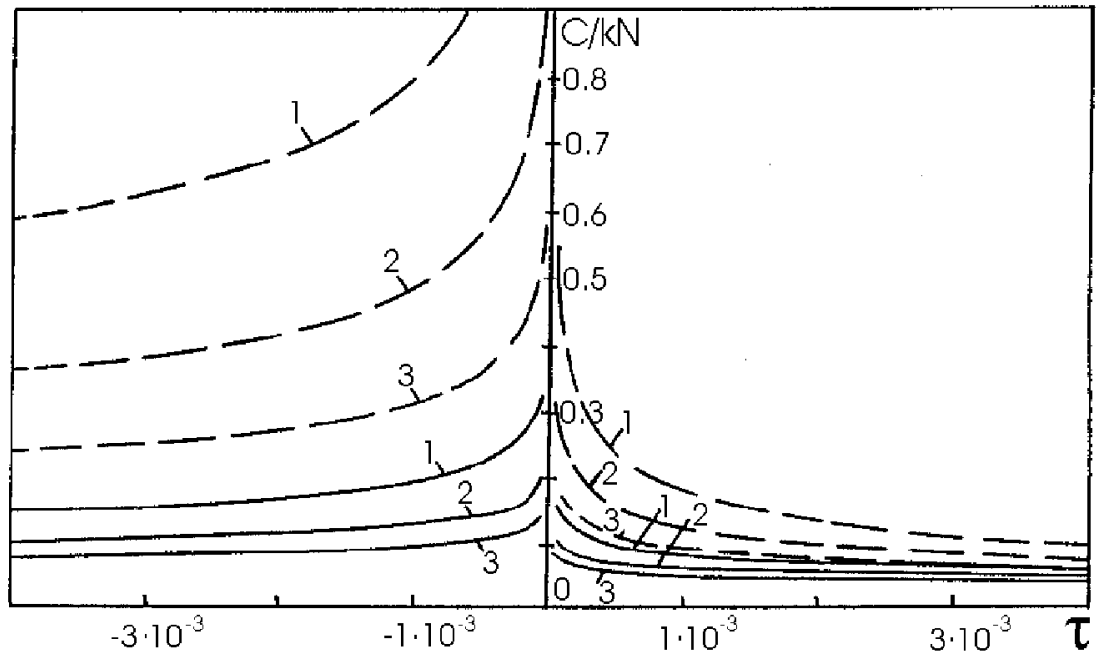

Figure 6. Specific heat of the system (Notations are the same as in figure 4).

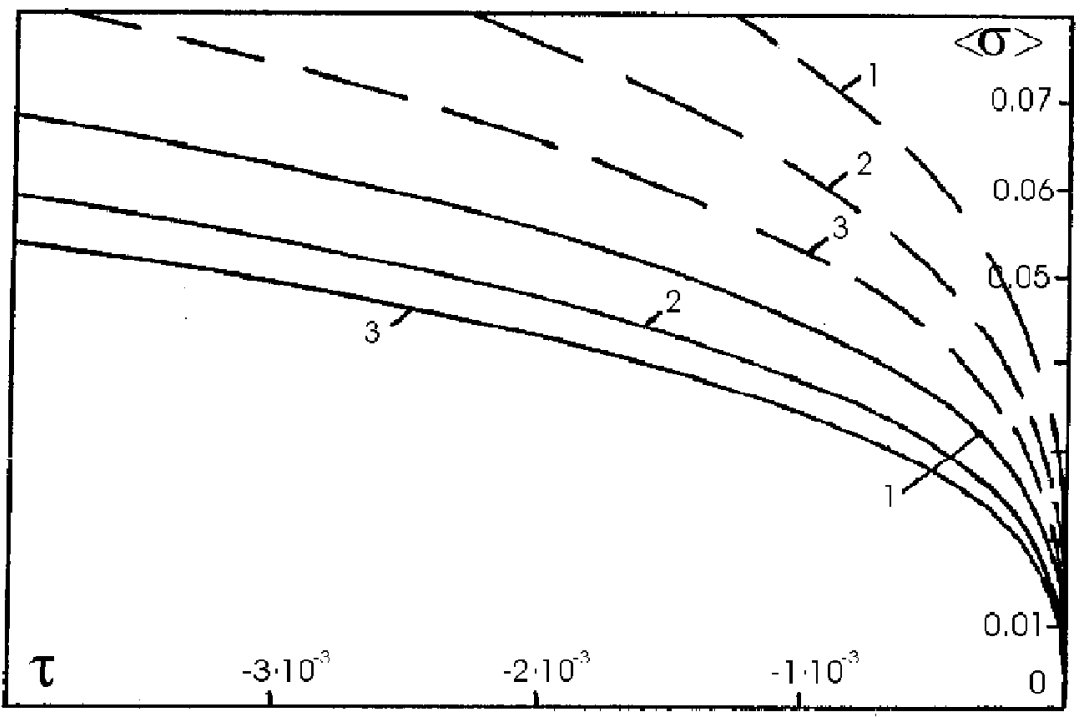

Figure 7. Mean spin moment $\langle\sigma\rangle$ at $T \leqslant T_{c}$ (Notations are the same as in figure 4$)$. 


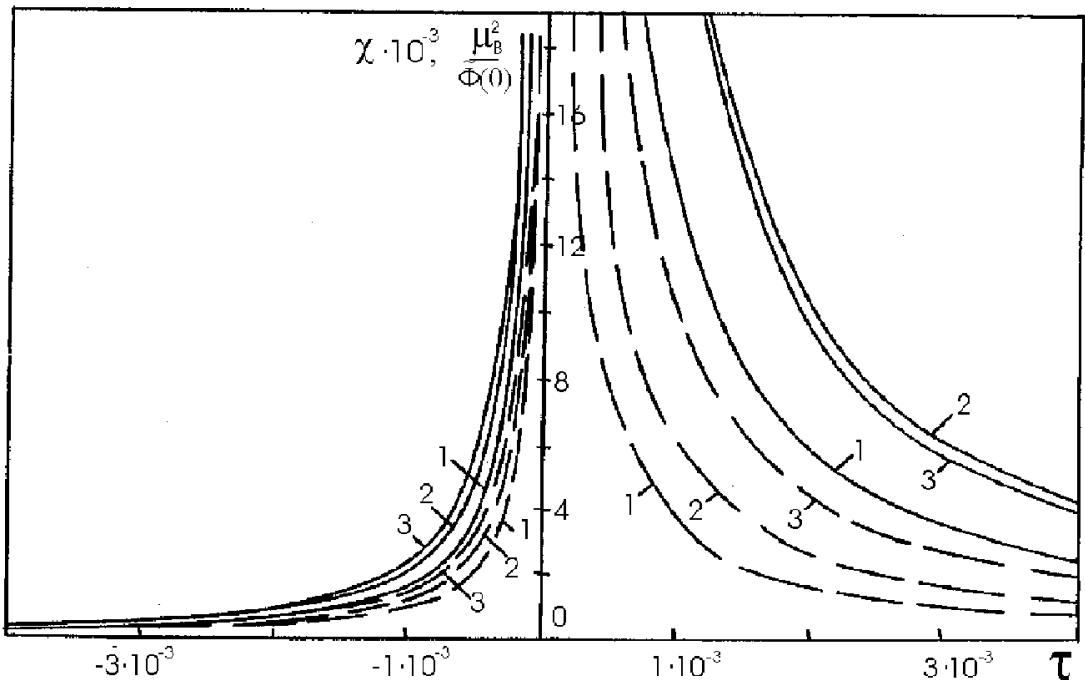

Figure 8 . Temperature dependence of the susceptibility $\chi$ (Notations are the same as in figure 4).

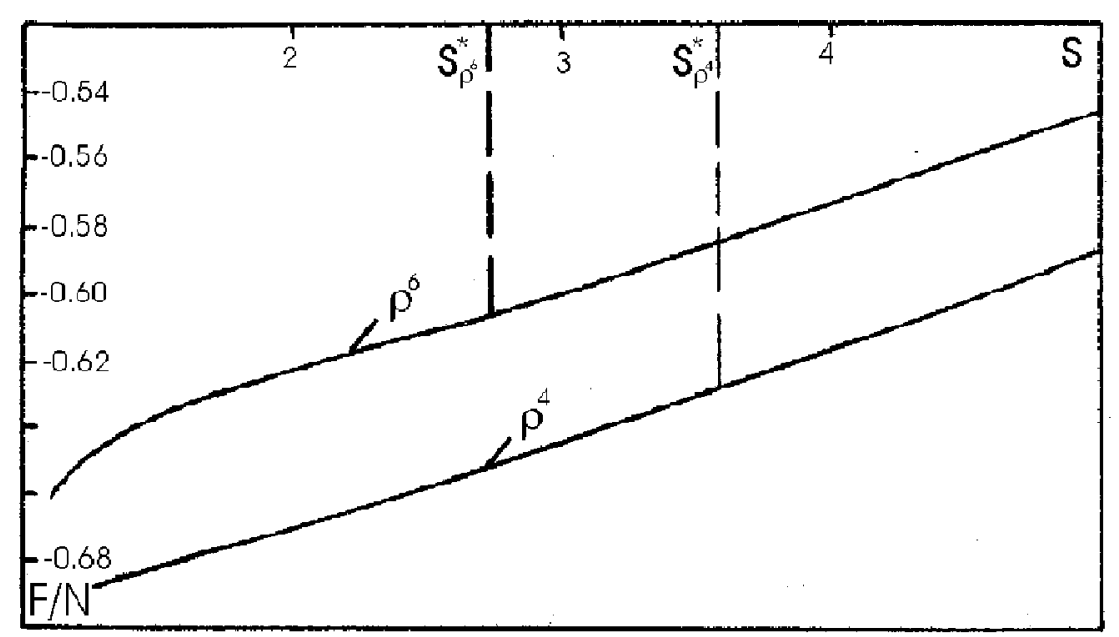

Figure 9. Behaviour of $F / N$ as a function of $s$ for $\rho^{4}$ and $\rho^{6}$ models. 
The corresponding plots of the thermodynamic characteristics calculated within the $\rho^{4}$ model, with the confluent corrections [9] being taken into account, are also given here (dashed curves). Comparison of these plots shows that the dependence of the thermodynamic functions on the parameter $s$ for the $\rho^{6}$ model is weaker than for the $\rho^{4}$ model. The dependence of $F / N$ on $s$ for these two models is represented in figure 9 .

Let us note that the calculations performed are best suited for the intermediate values of $s$, close to the quantity $s^{*}$, at which $h_{n}$ turns to zero at the fixed point $\left(s^{*}=2.7349\right.$ for the $\rho^{6}$ model and $s^{*}=3.5862$ for the $\rho^{4}$ model). The use of the difference form of the RR based on a non-Gaussian measure density works especially well for this region of $s$. At small values of $s(s \rightarrow 1)$, some complications arise when the unit element is extracted. In this limit, the RR should be represented as the perturbation series with respect to the Gaussian distribution ( $h_{n}$ is large at $s \rightarrow 1$, and expansions in $h_{n}^{-2}, \alpha_{n}$ can be used $\left.[19,20]\right)$. There also exists an upper limit for $s$. At large $s$, one must take into account the correction due to the potential averaging $[10,21]$, which increases with $s$.

The point $s \approx s^{*}$ corresponds to the beginning of the $\nu(s)$ curve stabilization [3]. Table 10 contains the values of the critical exponents $\nu, \alpha, \beta, \gamma$, the exponent of the scaling correction $\Delta_{1}=-\ln E_{2} / \ln E_{1}$, and the ratios of the critical amplitudes $A^{+} / A^{-}, \Gamma^{+} / \Gamma^{-}$and their combinations $P=\frac{1}{\alpha}[1-$ $\left.\frac{A^{+}}{A^{-}}\right], R_{c}^{+}=A^{+} \Gamma^{+} /\left[s_{0}^{3}\left(\langle\sigma\rangle^{(0)}\right)^{2}\right]$ at $s=s^{*}$ for $\rho^{4}$ and $\rho^{6}$ models. These values are in agreement with the data obtained within the field theory approach (FTA) [22-27] and high-temperature series (HTS) [28-32].

Table 10. Values of the critical exponents, ratios of the critical amplitudes and their combinations for $s=s^{*}$ $\left(s^{*}=2.7349\right.$ for the $\rho^{6}$ model and $s^{*}=3.5862$ for the $\rho^{4}$ model) obtained by means of the $\mathrm{CV}$ method. Data calculated within the field theory approach (FTA) [2227] and high-temperature series (HTS) [28-32].

\begin{tabular}{|c|c|c|c|c|}
\hline \multirow[b]{2}{*}{ Quantity } & \multicolumn{2}{|c|}{ Model } & \multicolumn{2}{|l|}{ Ref. data } \\
\hline & $\rho^{4}$ & $\rho^{6}$ & FTA & HTS \\
\hline$\nu$ & 0.605 & 0.637 & 0.630 & 0.638 \\
\hline$\alpha$ & 0.185 & 0.088 & 0.110 & 0.125 \\
\hline$\beta$ & 0.303 & 0.319 & 0.325 & 0.312 \\
\hline$\gamma$ & 1.210 & 1.275 & 1.241 & 1.250 \\
\hline$\Delta_{1}$ & 0.463 & 0.525 & 0.498 & 0.50 \\
\hline$A^{+} / A^{-}$ & 0.435 & 0.675 & $0.54,0.48$ & 0.51 \\
\hline$\Gamma^{+} / \Gamma^{-}$ & 6.967 & 9.253 & $4.77, \quad 5.12$ & 5.07 \\
\hline$P$ & 3.054 & 3.711 & $3.90,4.03,4.2,4.72$ & \\
\hline$R_{c}^{+}$ & 0.098 & 0.162 & $0.059,0.052$ & 0.059 \\
\hline
\end{tabular}




\section{Conclusions}

The method for the calculation of the three-dimensional Ising model thermodynamics is developed in the sixfold distribution approximation. Both temperature regions above and below the critical value of $T_{c}$ are considered. The main distinguishing feature of the approach is a separate allowance for contributions from the short- and long-wave fluctuation phases of the spin moment density to the free energy of the system near $T_{c}$. Within the framework of the $\rho^{6}$ model, we obtained the explicit expressions for the critical amplitudes of the thermodynamic functions of the three-dimensional Ising ferromagnet and calculated the coefficients of the free energy, the universal characteristics (the critical exponents, the ratios of the critical amplitudes). Calculation of the free energy, entropy, specific heat, mean spin moment, susceptibility was performed for different values of $s$. Comparison of the results obtained within $\rho^{4}$ and $\rho^{6}$ models indicates that the dependence of thermodynamic functions on the RG parameter $s$ is weaker for the $\rho^{6}$ model.

The values of $s$ close to $s^{*}$ are optimal for the method presented. Obtaining analytical expressions for the critical amplitudes and system thermodynamic characteristics as functions of the Hamiltonian microscopic parameters is the advantage of the method developed. The leading critical amplitudes for the specific heat and other thermodynamic characteristics are represented as a product of the universal part, independent of microscopic parameters, and the non-universal factor, which depends on these parameters.

\section{Acknowledgements}

This work was supported in part by the Ukrainian State Foundation of Fundamental Studies (project No 2.4/173). The authors are pleased to express their gratitude to this Foundation.

\section{References}

[1] Pylyuk I.V., Kozlovsky M.P. A study of the Ising model by using the nonGaussian basis measures. // Preprint ITP-87-31R, Inst. Theor. Phys., Acad. Sci. Ukr. SSR, Kiev, 1987, 28 p. (in Russian).

[2] Pylyuk I.V. The critical behaviour of the $3 \mathrm{D}$ one-component spin system in the collective variables method with the complication of the basis measure. // Preprint ITP-88-107R, Inst. Theor. Phys., Acad. Sci. Ukr. SSR, Kiev, 1988, 33 p. (in Russian).

[3] Kozlovsky M.P., Pylyuk I.V. Study of critical characteristics of the threedimensional Ising model by using the non-Gaussian density measures. // Ukr. Fiz. Zh., 1990, vol. 35, No 1, p. 146-147 (in Ukrainian).

[4] Kozlovsky M.P., Pylyuk I.V., Kolomiets V.A. The numerical investigation of the partition function of the Ising three-dimensional model using the sixfold basis distribution. // Preprint ITP-84-177R, Inst. Theor. Phys., Acad. Sci. Ukr. SSR, Kiev, 1984, 41 p. (in Russian).

[5] Kozlovsky M.P., Pylyuk I.V. Free energy and other thermodynamical functions above the second-order phase transition point. // Preprint ITP-85-23E, Inst. Theor. Phys., Acad. Sci. Ukr. SSR, Kiev, 1985, 48 p.

[6] Kozlovsky M.P., Ilnytsky J.M., Pylyuk I.V. Free energy and other thermodynamical functions of the three-dimensional Ising model below the phase transition point. // Preprint ITP-85-107R, Inst. Theor. Phys., Acad. Sci. Ukr. SSR, Kiev, 1985, 33 p. (in Russian). 
[7] Kozlovskii M.P., Pylyuk I.V. Calculation of the corrections to scaling for the thermodynamical functions of the Ising model. // Preprint ITP-89-24R, Inst. Theor. Phys., Acad. Sci. Ukr. SSR, Kiev, 1989, 29 p. (in Russian).

[8] Kozlovskii M.P., Pylyuk I.V. Comparison of the expressions for the thermodynamical functions of the Ising model at the temperatures higher and lower than the critical one in the three-dimensional space. // Preprint ITP-89-42R, Inst. Theor. Phys., Acad. Sci. Ukr. SSR, Kiev, 1989, 35 p. (in Russian).

[9] Pylyuk I.V. Thermodynamic functions of the 3D Ising ferromagnet in the vicinity of phase transition point with the first and second confluent corrections. // Preprint ITP-90-12R, Inst. Theor. Phys., Acad. Sci. Ukr. SSR, Kiev, 1990, 40 p. (in Russian).

[10] Yukhnovskii I.R. Phase transition of the second order. Collective variables method. Kiev, Naukova Dumka, 1985 (in Russian).

[11] Kozlovsky M.P. A critical behaviour of the three-dimensional Ising model. The model " $\rho "$ ". // Physics of Many-Particle Systems, 1983, iss. 4, p. 37-44 (in Russian).

[12] Kozlovsky M. Critical properties of Ising model. The $\rho^{6}$ model. General recurrent relations. // Preprint ITP-82-104R, Inst. Theor. Phys., Acad. Sci. Ukr. SSR, Kiev, 1982, 32 p. (in Russian).

[13] Kozlovskij M.P. The solutions of the renormalization group equations for Ising spin system in the $\rho^{6}$ model. // Preprint ITP-84-35R, Inst. Theor. Phys., Acad. Sci. Ukr. SSR, Kiev, 1984, 38 p. (in Russian).

[14] Kozlovsky M.P., Pylyuk I.V. Calculation of thermodynamic functions near phase transition point in six-fold basis measure approximation. // Preprint ITP-87-9R, Inst. Theor. Phys., Acad. Sci. Ukr. SSR, Kiev, 1987, 29 p. (in Russian).

[15] Kozlovskii M.P., Pylyuk I.V. Thermodynamics of the three-dimensional Ising ferromagnet in the vicinity of the phase transition point within the frames of $\rho^{6}$ model. Comparison with $\rho^{4}$ model. // Preprint ITP-90-81R, Inst. Theor. Phys., Acad. Sci. Ukr. SSR, Kiev, 1990, 53 p. (in Russian).

[16] Pylyuk I.V. Special functions for the investigation of critical properties of the three-dimensional Ising model within the frames of the sixth power measure density. // Ukr. Fiz. Zh., 1996, vol. 41, No 9, p. 885-894 (in Ukrainian).

[17] Yukhnovsky I.R. Solution of the three-dimentional Ising model in the temperature region below critical point. // Preprint ITP-83-85E, Inst. Theor. Phys., Acad. Sci. Ukr. SSR, Kiev, 1983, 39 p.

[18] Yukhnovsky I.R., Kozlovsky M.P., Shpot M.A. Free energy, entropy and heat capacity in the critical region at $T<T_{c}$. // Preprint ITP-84-144E, Inst. Theor. Phys., Acad. Sci. Ukr. SSR, Kiev, 1984, 40 p.

[19] Kozlovskii M.P., Ilnytskii Ja.N. Description of phase transition in systems with dimensionality close to four in the collective variables method. // Preprint ITP-87-100E, Inst. Theor. Phys., Acad. Sci. Ukr. SSR, Kiev, 1988, 17 p.

[20] Ilnytskii Ja.M., Kozlovskii M.P. The reexpansion procedure. Application to the asymptotic series of perturbation theory. // Preprint ITP-90-38U, Inst. Theor. Phys., Acad. Sci. Ukr. SSR, Kiev, 1990, 16 p. (in Ukrainian).

[21] Yukhnovskii I.R., Kozlovsky M.P., Pylyuk I.V. Correction for the potential averaging in the sequential method of the partition function calculation for the one-component spin system near $T_{c}$. // Preprint ITP-88-105R, Inst. Theor. Phys., Acad. Sci. Ukr. SSR, Kiev, 1988, 43 p. (in Russian).

[22] Le Guillou J.C., Zinn-Justin J. Critical exponents from field theory. // Phys. Rev. B, 1980, vol. 21, No 9, p. 3976-3998.

[23] Barmatz M., Hohenberg P.C., Kornblit A. Scaled-equation-of-state analysis of the specific heat in fluids and magnets near critical point. // Phys. Rev. B, 1975, vol. 12, No 5, p. 1947-1968.

[24] Singsaas A., Ahlers G. Universality of static properties near the superfluid transition in ${ }^{4} \mathrm{He}$.// Phys. Rev. B, 1984, vol. 30, No 9, p. 5103-5115.

[25] Bervillier C. Universal relations among critical amplitude. Calculations up to 
order $\epsilon^{2}$ for systems with continuous symmetry. // Phys. Rev. B, 1976, vol. 14, No 11, p. 4964-4975.

[26] Bagnuls C., Bervillier C., Meiron D.I., Nickel B.G. Nonasymptotic critical behavior from field theory at $d=3$. II. The ordered-phase case. // Phys. Rev. B, 1987, vol. 35, No 7, p. 3585-3607.

[27] Bagnuls C., Bervillier C., Boccara E. Estimates of universal combinations between thermodynamic critical amplitudes for Ising-like systems. // Phys. Lett., 1984, vol. 103A, No 9, p. 411-415.

[28] Gaunt D.S., Sykes M.F. Estimation of critical indices for the three-dimensional Ising model. // J. Phys. A, 1973, vol. 6, No 10, p. 1517-1526.

[29] Aharony A., Hohenberg P.C. Universal relations among thermodynamic critical amplitudes. // Phys. Rev. B, 1976, vol. 13, No 7, p. 3081-3090.

[30] Saul D.M., Wortis M., Jasnov D. Confluent singularities and the correctionto-scaling exponent for the $d=3 f_{c c}$ Ising model. // Phys. Rev. B, 1975, vol. 11, No 7, p. 2571-2578.

[31] Camp W.J., Van Dyke J.P. High-temperature series for the susceptibility of the spin-s Ising model: analysis of confluent singularities. // Phys. Rev. B, 1975, vol. 11, No 7, p. 2579-2596.

[32] Camp W.J., Saul D.M., Van Dyke J.P., Wortis M. Series analysis of corrections to scaling for the spin-pair correlations of the spin-s Ising model: confluent singularities, universality and hyperscaling. // Phys. Rev. B, 1976, vol. 14, No 9 , p. 3990-4001.

\section{МЕТОД РОЗРАХУНКУ ТЕРМОДИНАМІКИ ТРИВИМІРНОГО ІЗІНГІВСЬКОГО ФЕРОМАГНЕТИКА В РАМКАХ МОДЕЛІ $\rho^{6}$}

\footnotetext{
М.П.Козловський, І.В.Пилюк, В.В.Духовий

Здійснено розрахунок термодинамічних функцій тривимірного ізінгівського феромагнетика вище і нижче критичної температури в наближенні базисного розподілу, який включає шосту степінь змінної (модель $\rho^{6}$ ). Порівняння з результатами для моделі $\rho^{4}$ свідчить про послаблення залежності термодинамічних функцій від параметра ренормгрупи $s$. Вказаний оптимальний інтервал значень параметра ренормгрупи.
} 